ARTICLE

DOI: $10.1038 / \mathrm{s} 41467-018-04695-7$

OPEN

\title{
Soluble E-cadherin promotes tumor angiogenesis and localizes to exosome surface
}

Maggie K.S. Tang ${ }^{1}$, Patrick Y.K. Yue ${ }^{2}$, Philip P. Ip $\mathbb{D}^{3}{ }^{3}$, Rui-Lan Huang $\mathbb{1 0}^{4}{ }^{4}$, Hung-Cheng Lai ${ }^{4}$, Annie N.Y. Cheung ${ }^{3}$, Ka Yu Tse ${ }^{5}$, Hextan Y.S. Ngan $^{5}$ \& Alice S.T. Wong ${ }^{1}$

The limitations of current anti-angiogenic therapies necessitate other targets with complimentary mechanisms. Here, we show for the first time that soluble E-cadherin (sE-cad) (an 80-kDa soluble form), which is highly expressed in the malignant ascites of ovarian cancer patients, is a potent inducer of angiogenesis. In addition to ectodomain shedding, we provide further evidence that $\mathrm{sE}$-cad is abundantly released in the form of exosomes. Mechanistically, sE-cad-positive exosomes heterodimerize with $\mathrm{VE}$-cadherin on endothelial cells and transduce a novel sequential activation of $\beta$-catenin and NFKB signaling. In vivo and clinical data prove the relevance of $\mathrm{sE}$-cad-positive exosomes for malignant ascites formation and widespread peritoneal dissemination. These data advance our understanding of the molecular regulation of angiogenesis in ovarian cancer and support the therapeutic potential of targeting $\mathrm{sE}$-cad. The exosomal release of $\mathrm{sE}$-cad, which represents a common route for externalization in ovarian cancer, could potentially be biomarkers for diagnosis and prognosis.

\footnotetext{
${ }^{1}$ School of Biological Sciences, University of Hong Kong, Pokfulam Road, Pokfulam, Hong Kong. ${ }^{2}$ Department of Biology, Hong Kong Baptist University, Kowloon Tong, Hong Kong. ${ }^{3}$ Department of Pathology, University of Hong Kong, Sassoon Road, Pokfulam, Hong Kong. ${ }^{4}$ Department of Obstetrics and Gynecology, Shuang-Ho Hospital, Taipei Medical University, Taipei, Taiwan. ${ }^{5}$ Department of Obstetrics and Gynecology, University of Hong Kong, Sassoon Road, Pokfulam, Hong Kong. These authors contributed equally: Patrick Y. K. Yue, Philip P. Ip. Correspondence and requests for materials should be addressed to A.S.T.W. (email: awong1@hku.hk)
} 
T umor vasculature is an attractive therapeutic target. The fact that both the progressive growth of ovarian cancer and the formation of malignant ascites are critically dependent on angiogenesis suggests that anti-angiogenic therapeutic strategies may be meritorious to ovarian cancer treatment ${ }^{1,2}$. In particular, the burden of ascites as a complication in malignancy remains vitally important, as the extent of which is a characteristic of the aggressiveness and metastatic potential and a significant indicator of poor prognosis. Current peritoneocentesis is not effective in addressing the root cause of fluid accumulation and posing a significant risk of side effect ${ }^{3}$. Due to its central role in tumor angiogenesis, vascular endothelial growth factor (VEGF) has emerged as the most important angiogenic target. VEGF is overexpressed in most ovarian cancers. However, despite early clinical benefits in which VEGF has been targeted, most patients ultimately experience the development of resistance and disease progression, suggesting that other angiogenic regulators with complimentary mechanisms are needed ${ }^{4}$.

One of the hallmarks of metastatic progression is the dynamic regulation of cadherins (major cell-cell adhesion molecules) that play crucial roles in various aspects of the process, including cell growth, invasion, and migration ${ }^{5}$. Although E-cadherin is synthesized as a transmembrane molecule (a $120 \mathrm{kDa}$ glycoprotein), it can be cleaved off the ectodomain and released in a soluble form (sE-cad; $80-\mathrm{kDa}$ ), and this accounts for the decreased expression of functional E-cadherin at the cell surface ${ }^{6}$. This has been largely overlooked in the past because sE-cad can only be detected by examining protein size on western blots. Importantly, sE-cad is highly expressed in the serum and ascites of ovarian cancer $\left(6.18-89.56 \mu \mathrm{g} \mathrm{mL}^{-1}\right)$ and predicts a poor prognosis ${ }^{7}$. These observations underscore the importance of understanding the role of sE-cad in ovarian cancer. In general, sE-cad has only been considered in weakening cell-cell adhesion ${ }^{8}$. There is no information on whether sE-cad also has biological function itself which is critical for dictating metastatic spread. Moreover, the release of sE-cad has only been characterized in the mechanism of ectodomain shedding. While sE-cad has been found to be arisen from the tumor itself 9 , it is unclear whether there is other cleavage event.

Here, we show for the first time, in vitro and in vivo, that sE-cad is a pivotal regulator of angiogenesis. We also provide evidence that exosomes are a novel major platform for the cleavage and release of sE-cad in this process.

\section{Results}

sE-cad promotes HUVEC angiogenesis. Angiogenesis involves multiple steps, which include the disruption of the vasculature, cell migration, proliferation, and tube formation ${ }^{10}$. As such, we assayed for these activities to understand the mechanism of action. We first examined the endogenous level of sE-cad in three different human ovarian cancer cell lines (OVCAR-3, Caov-3, and OV-90). OVCAR-3, which possesses less or no metastatic potential, showed little expression of $\mathrm{sE}$-cad protein, whereas the protein was highly expressed in Caov-3 and OV-90, which have been shown to frequently metastasize when inoculated in mice ${ }^{11}$ (Supplementary Fig. 1a). The results also showed no sN-cad or sP-cad expression, except Caov-3, which also had high sP-cad content (Supplementary Fig. 1a). sE-cad, sN-cad, and sP-cad were absent in normal human ovarian surface epithelial (OSE) and fallopian tube epithelial (FTE) cells (Supplementary Fig. 1a, b). Caov-3 and OV-90, which had the highest sE-cad content of these cell lines analyzed, were used in subsequent experiments.

As shown, sE-cad was a potent stimulant of migration for human umbilical vein endothelial cells (HUVECs) (Fig. 1a). The use of an E-cadherin-neutralizing antibody against the ectodomain of E-cadherin HECD-1 to immunodeplete sE-cad from the conditioned media (Supplementary Fig. 2) resulted in diminished migration, confirming that the effect was $\mathrm{sE}$-cadspecific (Fig. 1a). Isotype-matched mouse IgG-treated sE-cad had no effect (Fig. 1a). A recombinant $\mathrm{Fc} / \mathrm{sE}$-cad chimera was migratory for HUVECs in the $\mu \mathrm{gL}^{-1}$ range, equivalent to the concentration present in the ascites (Supplementary Fig. 3a). Fc alone had no effect (Supplementary Fig. 3a). Neither conditioned media (Fig. 1b) nor Fc/sE-cad (Supplementary Fig. 3b) induced mitogenesis of HUVECs using the 3-(4,5-dimethylthiazol-2-yl)2,5-diphenyltetrazolium bromide-based cell viability assay, suggesting that sE-cad may not act as an endothelial cell mitogen. The breakdown of the vascular barrier has an important role in ascites formation and enhances metastasis ${ }^{12}$. To examine whether sE-cad induces endothelial barrier dysfunction, we performed in vitro permeability assay. Whereas under nonstimulated conditions, FITC-dextran flux did not occur across the monolayer, treatment of conditioned media (Fig. 1c) or Fc/sE-cad (Supplementary Fig. 3c) induced significant FITC-dextran flux. These results suggest that sE-cad mediates vascular permeability.

We next investigated the ability of sE-cad to promote the formation of three-dimensional capillary-like tubular structures of HUVECs on the basement membrane matrix mimics Matrigel, which encompasses all steps of angiogenesis ${ }^{13}$. As shown, conditioned media (Fig. 1d) or Fc/sE-cad (Supplementary Fig. 3d) caused a significant increase in tube formation, mimicking a physiological vasculature. These angiogenic effects were completely reversed by the addition of HECD-1-blocking antibodies, but not control IgG, indicating a critical role for sE-cad in the angiogenic phenotypes. Similar results were observed in human microvascular endothelial cells (HMVECs), which cover different parts of the vasculature, indicating that these effects were not restricted to HUVECs (Supplementary Fig. 4). In contrast, immunodepletion of sP-cad from the conditioned media of Caov-3, whereby sP-cad was most abundant, had no effect at all, showing that the angiogenic effect is specifically dependent on sEcad (Supplementary Fig. 5a, b). Consistent with previous observation ${ }^{14}$, Caov-3 and OV-90 expressed little or no VEGF. The VEGF-specific band could only be detected in concentrate conditioned media (30-fold) at the $\mathrm{pg} \mathrm{mL}^{-1}$ range (Supplementary Fig. 5c). VEGF could be selectively removed from the conditioned media by immunodepletion with the anti-VEGF antibody (Supplementary Fig. 5d) ${ }^{15}$. The addition of anti-VEGF, at a concentration of $10 \mu \mathrm{g} \mathrm{mL}^{-1}$, which was shown to completely block the mitogenic activity of $10 \mathrm{ng} \mathrm{mL}^{-1}$ VEGF in HUVECs ${ }^{16}$, did not cause significant alterations of migration and tube formation (Supplementary Fig. 5e, f). It is however possible that, in vivo, VEGF concentrations might reach higher levels due to diffusion constrains within intact tissues ${ }^{17}$. It has also been shown that extracellular processing by proteases is required to activate VEGF release ${ }^{16}$.

To determine whether sE-cad was angiogenic in vivo, we employed a Matrigel implant murine model. This model has been standardized extensively for studying morphological and functional neovascularization ${ }^{18}$. As shown in Fig. 1e and Supplementary Fig. 3e, Matrigel containing conditioned media or Fc/sE-cad revealed extensive angiogenesis in the implants that were corroborated with the in vitro results. The presence of intact red blood cells inside the neovessels indicated that they are functional (Fig. 1e and Supplementary Fig. 3e). In contrast, treatment of HECD-1 inhibited the sE-cad-induced neovascularization. Thus, sE-cad also has the capacity to induce angiogenesis in vivo.

sE-cad is secreted from ovarian cancer cells in exosomes. For both Caov-3 and OV-90, the presence of the $80 \mathrm{kDa}$ E-cadherin 
fragment corresponding to sE-cad production in the cytoplasm suggested that sE-cad released was composed of two forms: a membrane-cleaved sE-cad form and a full-length form in membrane vesicles (Fig. 2a). To enable a refined analysis of sE-cad release, we used sucrose gradient centrifugation. Interestingly, while E-cadherin was detected in the plasma membrane-enriched fractions in the bottom of the gradient, most $(\sim 70 \%)$ E-cadherin was predominantly expressed in Golgi/Trans-Golgi networkenriched fractions where E-cadherin was present and colocalized with the Golgi marker GM130 (Fig. 2b). The cytoplasmic fragment of E-cadherin $(38 \mathrm{kDa})$, possibly generated by cleavage of the full-length E-cadherin, was also observed (Fig. 2b). These results suggest that E-cadherin ectodomain cleavage occurs not only at the plasma membrane, but also in the Golgi/Trans-Golgi network.

Because a significant amount of sE-cad is found in the Golgi/ trans-Golgi network, we postulated that it might exist in microvesicles ${ }^{19}$. To test this assumption, the isolated vesicles
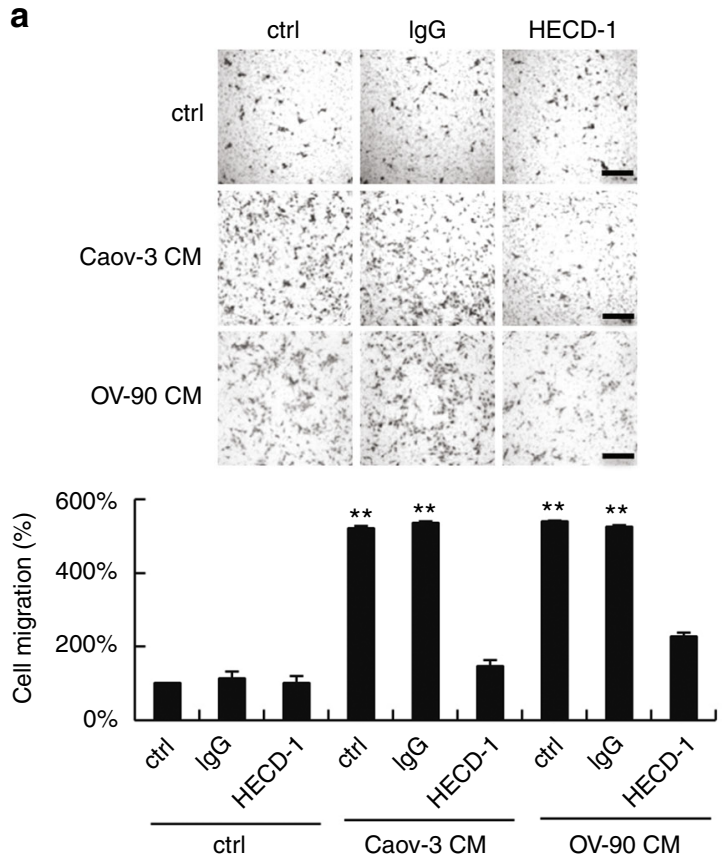

d
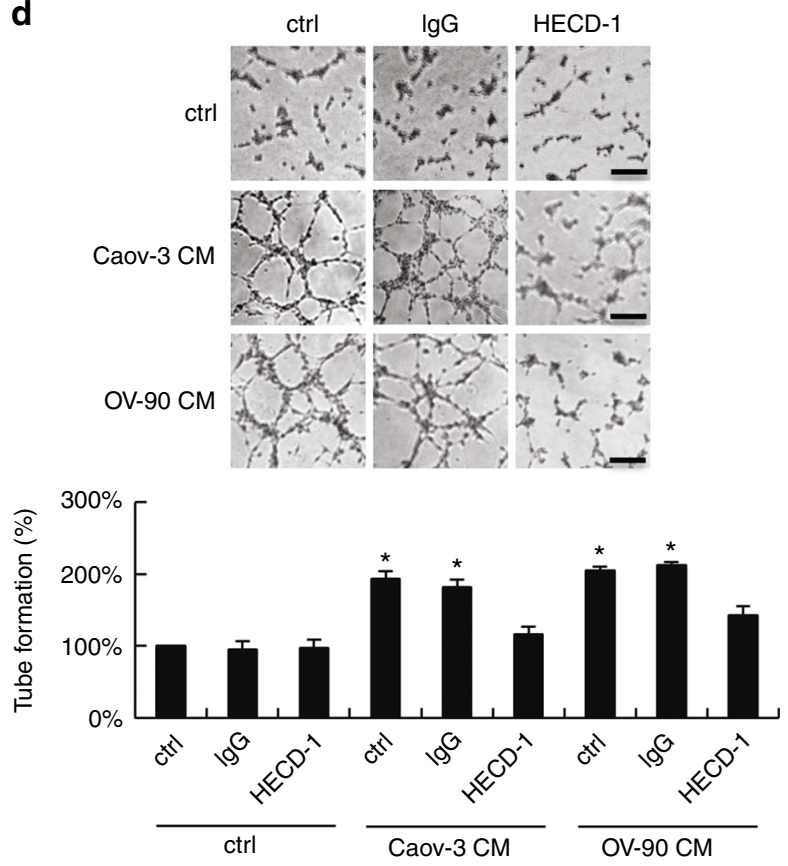

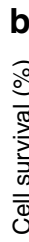
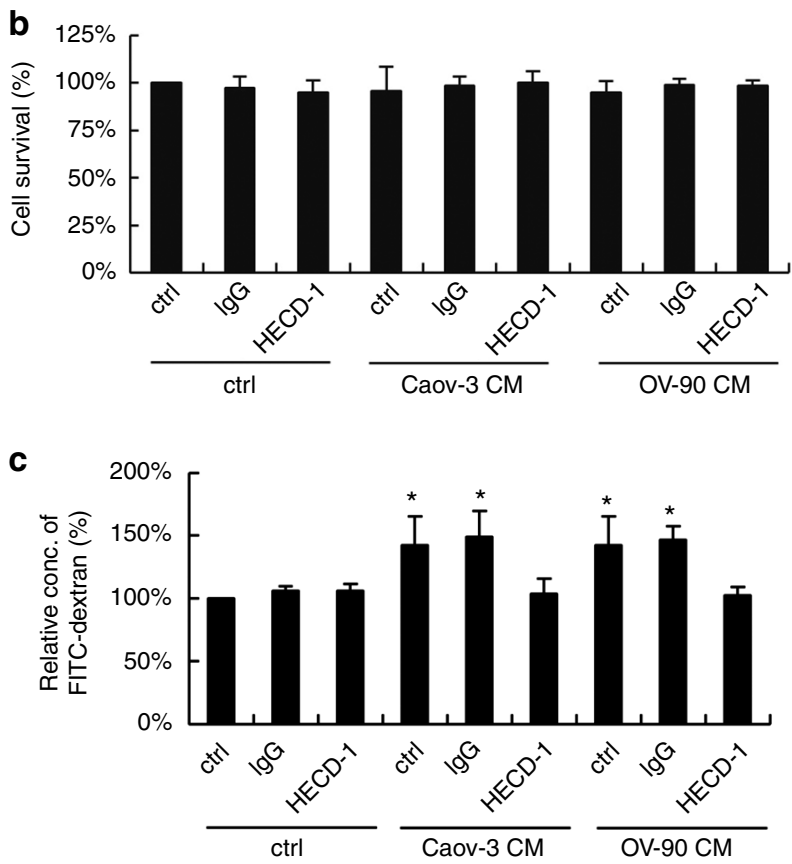

e
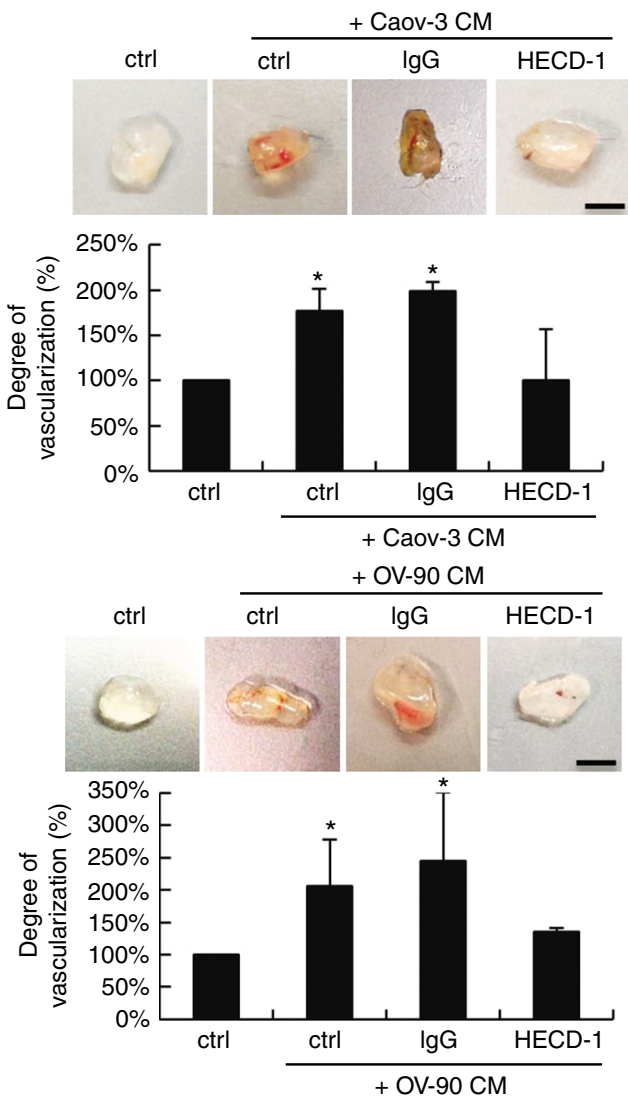
were analyzed with other exosomes markers, such as the tetraspan CD63 and Tsg101, that were also present in the isolated vesicles (Fig. 2c). To exclude potential interference of the subcellular fractions, we performed analogous experiments with GRP78 (endoplasmic reticulum marker), cytochrome c (mitochondria marker), and nucleoporin p62 (nuclear envelope marker) that confirmed their absence in the isolated fractions of the gradient (Fig. 2c). As revealed by sucrose density centrifugation, sE-cad containing vesicles floated in the middle of the gradient of density $1.1036-1.1612 \mathrm{~g} \mathrm{~cm}^{-3}$ positive for Hsp70, an established marker for exosomes (Fig. 2d). Electron microscopy analysis of the sucrose step gradient fractions identified disc-shaped vesicles with a diameter of around 100 $\mathrm{nm}$ in the fractions, typical of exosomes $(50-150 \mathrm{~nm})^{20}$ (Fig. 2e). Localization of sE-cad was also documented by immunoelectron microscopy on the exosomal surface (Fig. 2e). CD63, commonly identified on the surface, was included as a positive control (Fig. 2e). No staining was detected on exosomes stained with control antibody (Fig. 2e). Nanoparticle tracking analysis was performed using ZetaView and revealed $2.4 \times 10^{7}$ particles per $\mu \mathrm{g}$ of Caov-3 exosomes with a median diameter of $117.8 \mathrm{~nm}$ and $4.1 \times 10^{7}$ particles per $\mu \mathrm{g}$ of OV-90 exosomes with a median diameter of $122.8 \mathrm{~nm}$ (Fig. 2f).

sE-cad-positive exosomes promote angiogenesis. Next, we investigated the putative angiogenic activity of $\mathrm{sE}$-cad-positive exosomes. As shown in Fig. 3, sE-cad-positive exosomes were a potent inducer of angiogenesis. The enhanced endothelial cell migration (Fig. 3a), permeability (Fig. 3b), and tube formation (Fig. 3c), as well as neovascularization in Matrigel implants (Fig. 3d), were blocked in the presence of $\mathrm{mAb}$ to E-cadherin. These results are in agreement with above studies, which showed that expression of sE-cad in conditioned media or Fc/sE-cad was sufficient to induce angiogenesis. Next, sE-cad-positive exosomes were labeled with a BODIPY-TR ceramide fluorescent dye. We showed that the labeling occurred on HUVECs, which could be reverted by treatment with HECD-1, suggesting the uptake of exogenous purified sE-cad-positive exosomes by HUVECs (Fig. 3e). In contrast, no labeling was observed in the control sample (Fig. 3e). Interestingly, in line with a dose-dependent angiogenic response as shown in conditioned media (Supplementary Fig. 6a) or Fc/sE-cad (Supplementary Fig. 6b), OV-90, which expresses high levels of sE-cad-positive exosomes (Supplementary Fig. 6c), showed much increased angiogenic responses than in Caov-3 (Fig. 3d). These results were corroborated with HUVECs treated with sE-cad-positive exosomes at doses 10-50 $\mu \mathrm{g} \mathrm{mL} L^{-1}$, which caused a significant increase in endothelial tube formation (Supplementary Fig. 6d). In addition, sE-cad-positive exosomes demonstrated a similar angiogenic response in endothelial cell migration and tube formation even at a protein concentration four times lower than sE-cad proteins from conditioned media (Supplementary Fig. 7). sE-cad-positive exosomes also gave an angiogenic effect similar to that of a clinically relevant dose (10 $\left.\mathrm{ng} \mathrm{mL}^{-1}\right)$ of VEGF in malignant ascites (Supplementary Fig. $8 \mathrm{a})^{21}$, demonstrating potent angiogenic efficacy of sE-cad-positive exosomes. In addition, a specific blocking antibody against VEGF shown to block VEGF-induced endothelial tube formation had no effect on sE-cad-positive exosomemediated tube formation by these HUVECs (Supplementary Fig. 8a). To further investigate any role of sE-cad in regulating VEGF, we examined the effect of sE-cad-positive exosomes on VEGF expression. As shown in Supplementary Fig. 8b, sE-cadpositive exosomes showed no effect on VEGF expression. Moreover, when combined, sub-threshold doses of sE-cadpositive exosomes and VEGF significantly promoted endothelial tube formation $(1.4$-fold $)(P<0.05)$, at which neither dose of sEcad-positive exosomes nor VEGF alone significantly affected endothelial tube formation (Supplementary Fig. 8c), consistent with the VEGF independence that we observed.

VE-cadherin as $\mathbf{s E - c a d}$ binding protein. Because HUVECs do not express E-cadherin, other cell surface molecule may be involved. For this, we carried out affinity chromatography using His-tagged Fc/sE-cad chimera on Ni-NTA beads. We identified VE-cadherin as a putative sE-cad binding protein (but not other cadherins or integrins) on HUVEC surface (Fig. 4a). To obtain evidence for the direct binding of sE-cad to VE-cadherin, HUVECs were treated with Caov-3-derived exosomes, immunoprecipitated with anti-VEcadherin, and then subjected to immunoblotting with anti-Ecadherin and anti-VE-cadherin antibodies. As shown in Fig. 4b, cell surface-bound E-cadherin was readily detected. In the presence of anti-VE-cadherin, the effect of $\mathrm{sE}$-cad-positive exosomes on angiogenesis by enhancing endothelial cell tube formation was clearly abrogated (Fig. 4c), indicating that sE-cad acts through a VE-cadherin-dependent mechanism and induces angiogenesis.

\section{sE-cad-positive exosomes mediate angiogenesis via $\beta$-catenin.} $\beta$-catenin and p120 catenin associate with the intracellular domain of cadherins, promote both cell-cell adhesion and cell signaling 22 . The most important aspect of these catenins is the balance between its cadherin-bound forms and the nuclear pool; only nuclear $\beta$-catenin and p120 catenin can affect cell signaling activity. We did western blot analysis to examine the subcellular distribution of $\beta$-catenin and p120 catenin in HUVEC in response to exosomes, and found that $\beta$-catenin accumulated extensively in the nuclei upon $30 \mathrm{~min}$ stimulation and was maintained even 60 min later (Fig. 4d). The amounts of p120 catenin protein estimated by western blotting were the same (Fig. 4d). Nuclear $\beta$-catenin was specific, as the signal was lost after HECD-1 mediated inhibition of sE-cad (Fig. 4e). Importantly, $\beta$-catenin knockdown significantly inhibited angiogenic tube formation in the presence of sE-cad-positive exosomes

\footnotetext{
Fig. $1 \mathrm{sE-cad} \mathrm{promotes} \mathrm{angiogenesis} \mathrm{in} \mathrm{vitro} \mathrm{and} \mathrm{in} \mathrm{vivo.} \mathrm{a} \mathrm{Cell} \mathrm{migration} \mathrm{assay} \mathrm{of} \mathrm{HUVEC} \mathrm{treated} \mathrm{with} \mathrm{control} \mathrm{(ctrl)} \mathrm{or} \mathrm{immunodepleted} \mathrm{conditioned}$ medium (CM) of Caov-3 and OV-90. Upper: Representative images of HUVEC migration. Lower: Quantification of the percentage change of the number of migrated cells. Bar, $100 \mu \mathrm{m}$. b Proliferation assay of HUVEC treated with control (ctrl) or immunodepleted conditioned medium (CM) of Caov-3 and OV90 in the presence or absence of E-cadherin neutralizing antibodies, HECD-1 $\left(100 \mu \mathrm{g} \mathrm{mL}^{-1}\right)$. c Permeability analysis of HUVEC measured by the percentage change of FITC-dextran flux (excitation $485 \mathrm{~nm}$, emission $535 \mathrm{~nm}$ ) treated with control (ctrl) or immunodepleted conditioned medium (CM) of Caov-3 and OV-90. d Tube formation assay of HUVEC treated with control (ctrl) or immunodepleted conditioned medium (CM) of Caov-3 and OV-90. Upper: Representative images of HUVEC tube formation assay. Lower: Quantification of the percentage change of the number of branching points. Bar, $100 \mu \mathrm{m}$. $\mathbf{e}$ In vivo Matrigel plug implant model using C57/BL6 mice subcutaneously injected with control (ctrl) or immunodepleted conditioned medium (CM) of Caov-3 and OV-90. In vivo neovascularization is measured by the Drabkin's reagent kit after 7 days. Upper: Representative images of excised Matrigel plug. Lower: Quantification of the percentage change in hemoglobin content. Bar, $5 \mathrm{~mm}$. For in vivo Matrigel plug model, $n=6$ per group, and were conducted twice. For the other assays, $n=3$ per group, all experiments were repeated three times. Error bar indicates SD of the mean. ${ }^{\star} P<0.05,{ }^{\star \star} P<0.01$ versus untreated control using one-way analysis of variance followed by Tukey's least significant difference post hoc test
} 
(Fig. 4f). Treatment of HUVEC with anti-VE-cadherin showed similar, much inhibited nuclear $\beta$-catenin responses to sE-cadpositive exosomes (Fig. $4 \mathrm{~g}$ ), suggesting that $\beta$-catenin is critically involved in the sE-cad/VE-cadherin-mediated heterodimerization that contributes to increased angiogenesis.

a

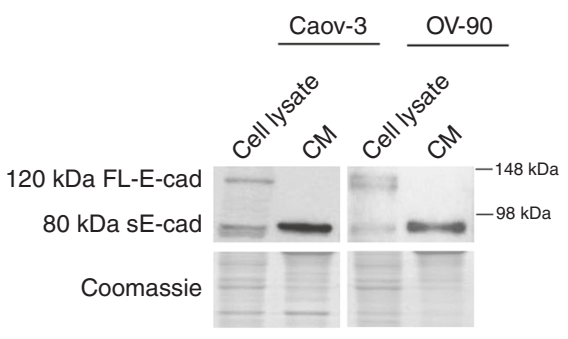

C

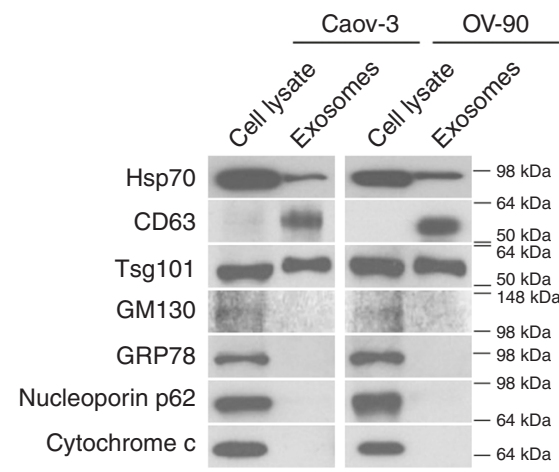

d

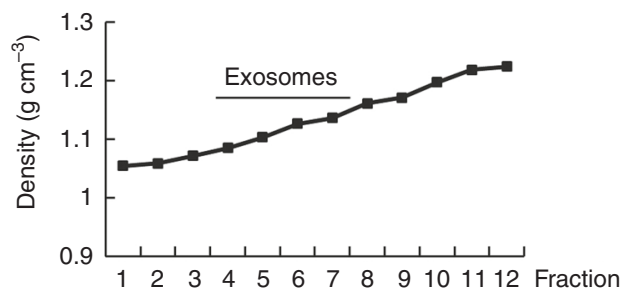

Caov-3

Exosomes

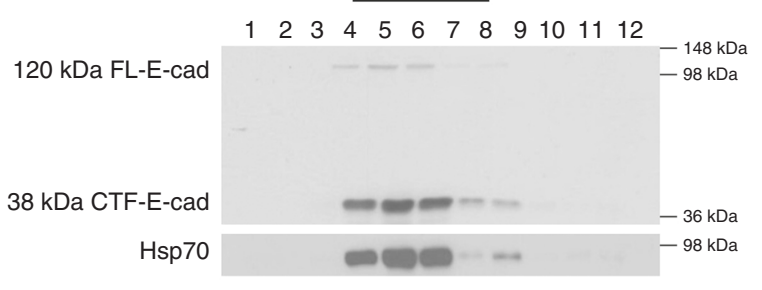

OV-90

Exosomes

$\begin{array}{llllllllllll}1 & 2 & 3 & 4 & 5 & 6 & 7 & 8 & 9 & 10 & 11 & 12\end{array}$

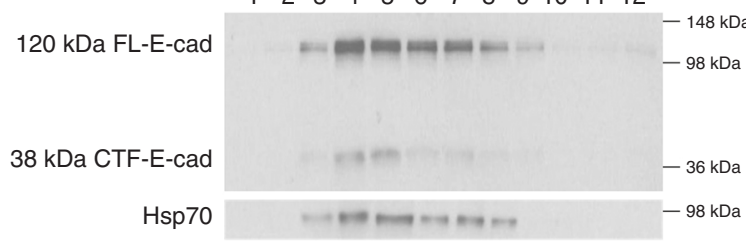

sE-cad-positive exosomes activate the NFkB signaling cascade. To investigate novel signaling paths downstream of sE-cad-VEcadherin in HUVECs, we compared sE-cad-positive exosomestreated HUVECs cultured with or without HECD-1 using Affymetrix gene chips Human Gene 2.0 ST, which covers over 30,000

b

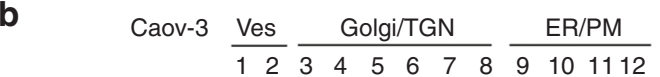
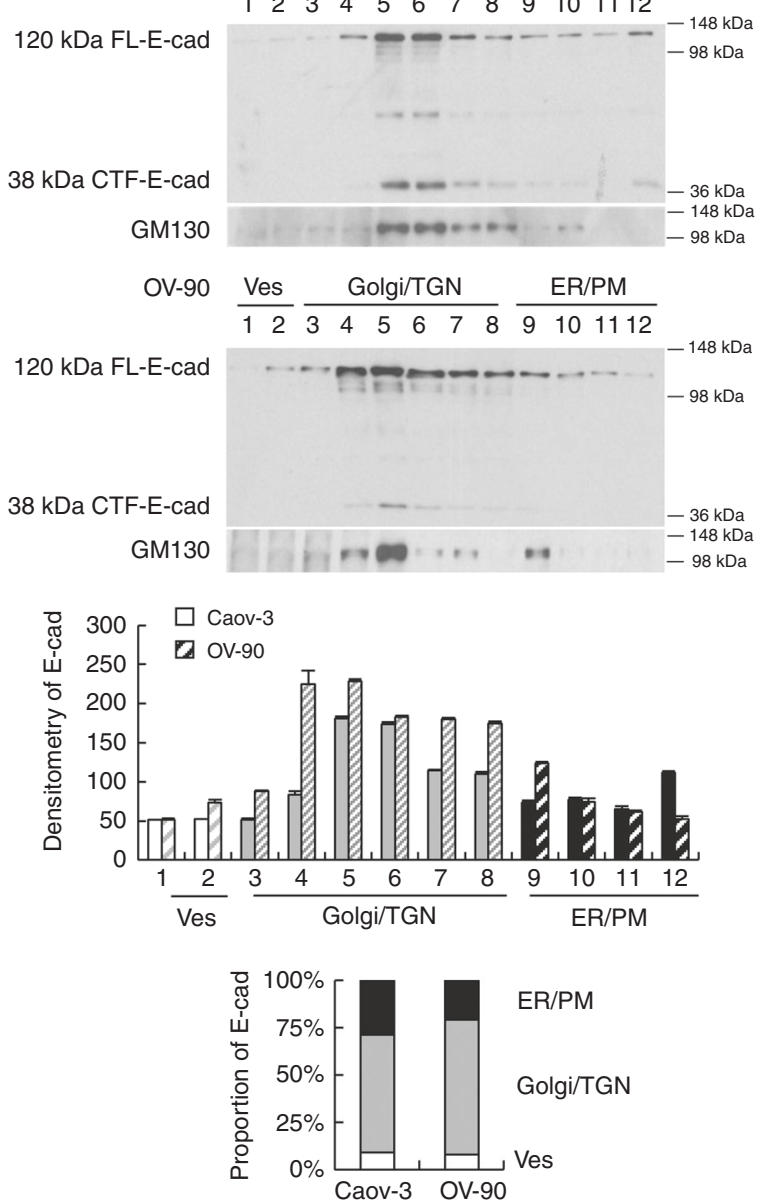

e

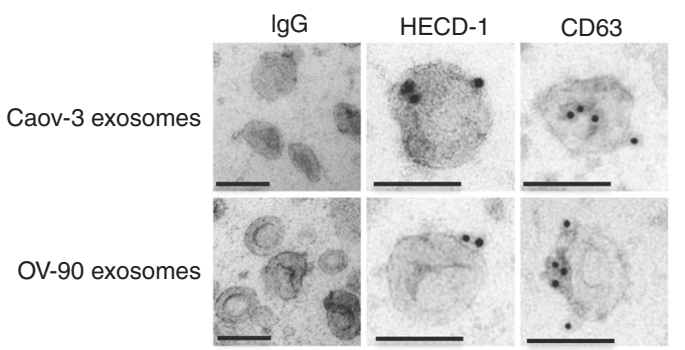

f

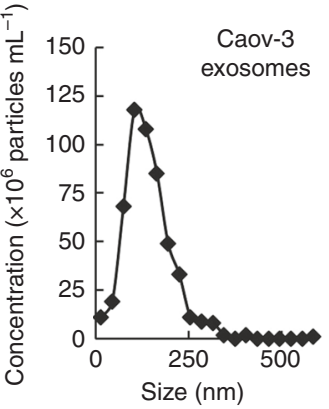


coding transcripts. 840 different genes were significantly upregulated and 691 were significantly downregulated by at least 1.5 fold (Fig. 5a). The genes were subjected to ingenuity pathway analysis (IPA), in which NFKB is in the central position of these networks (Fig. 5a). The altered transcriptional activities of NFkB in response to $\mathrm{sE}$-cad-positive exosomes and the interaction with VE-cadherin were confirmed using luciferase reporter assays (Fig. 5b). NFkB activation cascade is characterized by the nuclear translocation of $\mathrm{p} 65 / \mathrm{p} 50$ or $\mathrm{RelB} / \mathrm{p} 52$ subunits $^{23}$. Western blot analysis of the nuclear extract of HUVECs identified p65/p50 as the key player with sE-cad-positive exosomes (Fig. 5c). The NFkB inhibitor, Bay11-7082, potently inhibited angiogenic tube formation in the presence of $\mathrm{sE}$-cad-positive exosomes (Fig. $5 \mathrm{~d}$ ). These results indicate that $\mathrm{NF \kappa B}$ contributes to the angiogenic behavior of sE-cad-positive exosomes and suggest that activity could be mediated through the classical NFkB pathway. Several studies provide evidence for interactions between the $\beta$-catenin and NFKB signaling pathways ${ }^{23-25}$. By using coimmunoprecipitation studies, we did not detect $\beta$-catenin in the p65 coimmunoprecipitated complex nor in the reciprocal experiment in both control and sE-cad-positive exosome-treated HUVECs (Fig. 5e). Moreover, knocking down $\beta$-catenin by siRNA had no effect on NFkB-dependent transcriptional activities (Fig. 5f). Nor did p105/ p50 and p65 siRNA affect $\beta$-catenin/TCF-dependent transcriptional activities (Fig. 5f). Interestingly, as we followed the time course of the status of these two pathways, we found that $\beta$ catenin was activated and already at its maximum by $30 \mathrm{~min}$. On the contrary, nuclear translocation of NFKB was not noted until $120 \mathrm{~min}$ (Fig. 5g). The downregulation of either $\beta$-catenin or NFkB by siRNA could abolish the sE-cad-positive exosomesinduced angiogenic tube formation, whereas the inhibition of both $\beta$-catenin and NFKB had no additive effects (Fig. 5h). These data suggest that these two pathways may signal through sequential and independent cascades.

sE-cad-positive exosomes trigger angiogenesis in vivo. To determine whether the in vitro effect on angiogenesis was recapitulated in vivo, sE-cad-negative HEYA8 (Supplementary Fig. 1c) tumor-bearing mice were treated intraperitoneally twice weekly with IgG or HECD-1 pretreated OV-90-derived exosomes for 21 days. OV-90-derived exosomes induced a dose-dependent increase in the number of disseminated tumor nodules and ascites formation, which was noted at $5 \mu \mathrm{g}$ and significant at 15 and 25 $\mu \mathrm{g}$ per dose (Supplementary Fig. 6e). Treatment with HECD-1 significantly delayed both the number of disseminated tumor nodules and ascites formation compared with IgG control mice (Fig. 6a). In addition, HECD-1 treatment led to a marked decrease in neovascularization, which was characterized by newly formed immature vessels, indicating a role for sE-cad-positive exosomes in ovarian tumor angiogenesis (Fig. 6b). CD31 staining also revealed a decrease in tumor microvessel density (MVD) (Fig. 6b). Additionally, sE-cad-positive exosomes produced a marked increase in extravasation of Evans blue, which indicates albumin and plasma protein leakage into the interstitial tissue ${ }^{26}$ (Fig. 6c). Treatment with HECD-1 resulted in a significant inhibition of tumor vessel leakage (Fig. 6c). Next, we isolated vesicles from the ascitic fluids of ovarian carcinoma patients $(n=$ 35). As shown in Fig. 6d, 25 out of 35 ascites contained sE-cad and sedimented in the middle of the gradient equivalent to the density of exosomes, confirming that the cleavage of E-cadherin in released vesicles that we showed in cell cultures might be found in situ in ovarian carcinomas. This finding would also suggest that exosomes may be a factor responsible for the release of $\mathrm{sE}$ cad in vivo. Expression of sE-cad-positive exosomes was defined as high $(+++)\left(>20 \mu \mathrm{g} \mathrm{mL}^{-1}\right)$, medium $(++)\left(10-20 \mu \mathrm{g} \mathrm{mL}^{-1}\right)$, low $(+)\left(<10 \mu \mathrm{g} \mathrm{mL}^{-1}\right)$, and none $(-)\left(0 \mu \mathrm{g} \mathrm{mL}^{-1}\right)$ levels. Our analyses revealed high levels of $\mathrm{sE}$-cad-positive exosomes $\left(2.62-27.10 \mu \mathrm{g} \mathrm{mL}^{-1}\right)$ in the ascitic fluids of ovarian carcinomas (Supplementary Table 1). There was no strong association between the expression levels of sE-cad-positive exosomes and tumor histologic subtypes (Supplementary Table 1), which were consistent with those of the cell lines (Supplementary Fig. 9). In contrast, peritoneal fluids of non-cancerous (benign) patients exhibited no or only weak $\left(3.92-8.13 \mu \mathrm{g} \mathrm{mL}^{-1}\right)$ expression of sEcad-positive exosomes (Supplementary Table 2). In accord with the ovarian cancer cell lines data, patient ascites-derived exosome was a potent inducer of endothelial tube formation (Fig. 6f). The enhanced number and length of capillary tubes formed by endothelial cells were blocked in the presence of the $\mathrm{mAb}$ to Ecadherin (HECD-1), but not to nonimmune IgG (Fig. 6f). Moreover, treatment with patients'-derived exosomes substantially augmented ascites formation, tumor dissemination, and neovascularization as compared with the control group, and that the effects were abrogated in the presence of HECD-1 (Fig. 6g, h), further supporting the rationale of therapeutic targeting of $\mathrm{sE}$ cad.

\section{Discussion}

It is well recognized that tumor cells can modify their microenvironment using proteolytes ${ }^{27}$. The critical question is whether the generation of $\mathrm{sE}$-cad is merely a byproduct of tumor cells or offers advantages to the tumor cells. In the present study, we show for the first time sE-cad is a potent inducer of angiogenesis. Notably, we also provide evidence for an important role of extracellular vesicles in the constitutive release of sE-cad from tumor cells both in vitro and in vivo. As exosomes isolated from ovarian cancer patients ascites are also functionally active, our findings provided new insight into the clinical correlation between levels of sE-cad in ascites and the advanced stage of ovarian cancer.

We demonstrated that sE-cad stimulates (a) endothelial cell migration, (b) permeability, (c) endothelial cell tube formation, and (d) neovascularization in Matrigel implants. However, it does not act as an endothelial cell mitogen, which has been demonstrated for cell adhesion molecules such as ICAM-1, E-selectin, and VCAM-1 28,29 . These findings can be interpreted in light of their potent migration potential, which is most closely associated with the angiogenic function, may be responsible for its angiogenic activity ${ }^{30}$.

Fig. 2 Intracellular cleavage of sE-cad and the release of sE-cad-positive exosomes. a Western blot analysis of E-cadherin in total cell lysate and conditioned medium (CM) of Caov-3 and OV-90. b Western blot analysis of the subcellular localization of E-cadherin in Caov-3 and OV-90 by sucrose density gradient fractionation. Ves vesicles, TGN trans-Golgi network, ER endoplasmic reticulum, PM plasma membrane. Upper: Representative western blot images. Lower: Densitometry of FL-E-cad. c Western blot analysis of the protein composition of exosomes isolated from Caov-3 and OV-90. d Sucrose density gradient fractionation of exosomes isolated from Caov-3 and OV-90. Upper: Densities ( $\mathrm{g} \mathrm{cm}^{-3}$ ) of each sucrose fraction as determined by refractometry. Lower: Representative images of western blot. e Immunoelectron microscopic view of the sE-cad localization on the exosomal surface of Caov-3 and OV-90 cells at a magnification of 52,000×. Bar, $100 \mathrm{~nm}$. Localization of CD63 as a positive control was visualized. No staining was found on exosomes incubated with an isotype-matched control lgG. $\mathbf{f}$ The number of particles per $\mu$ g exosomes and median diameter were determined by nanoparticle tracking analysis. $n=3$ per group, all experiments were repeated three times. Error bar indicates SD of the mean 
a

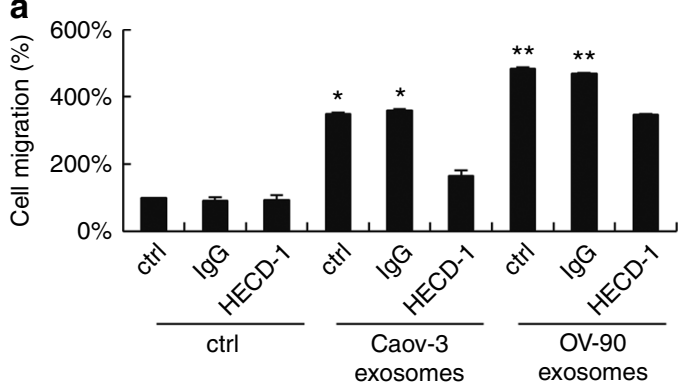

C

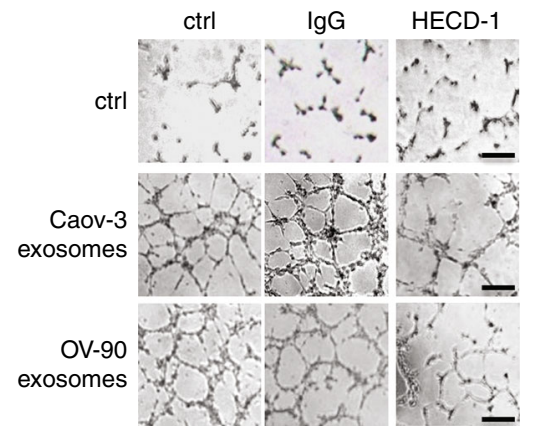

d
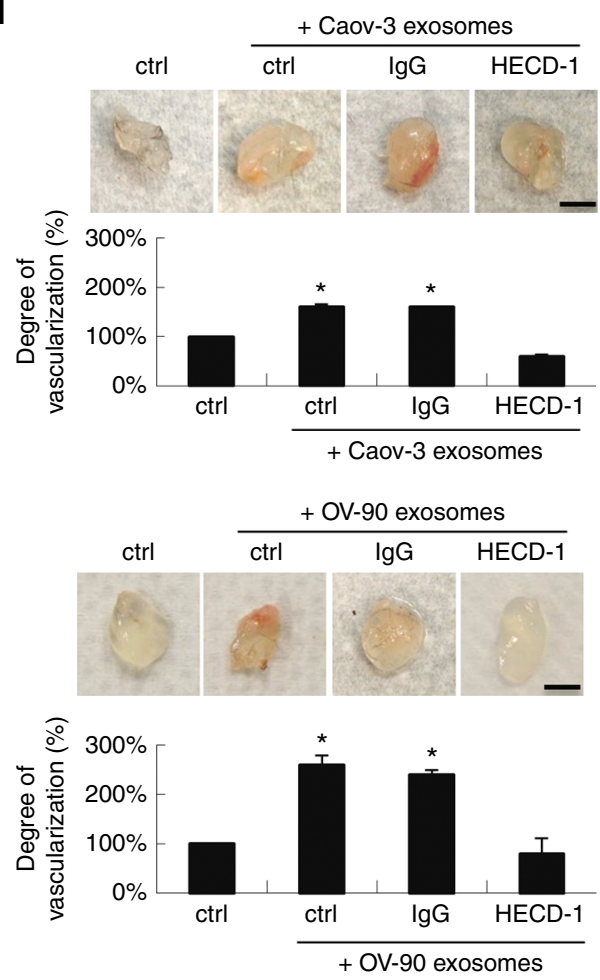

b
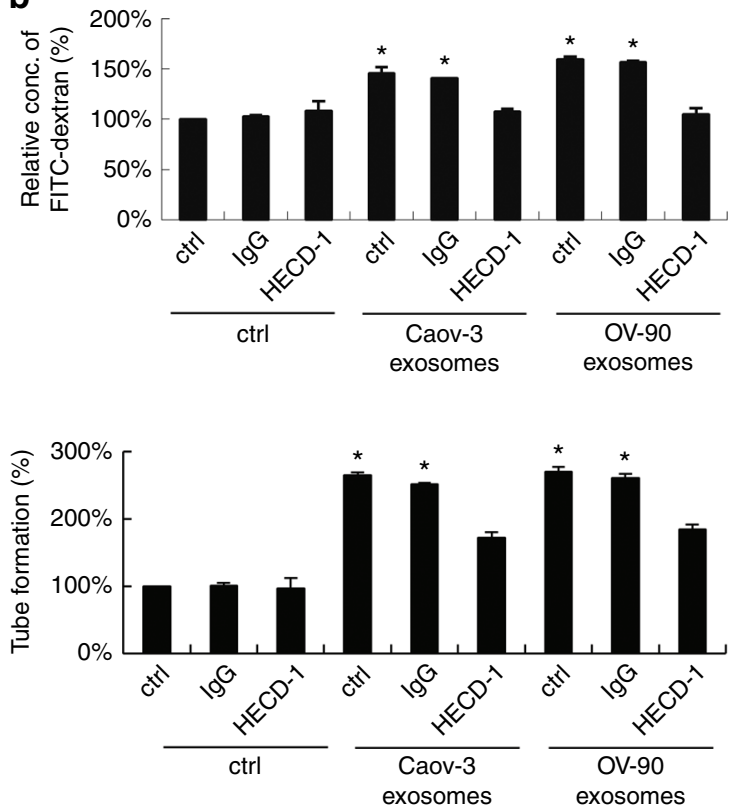

e

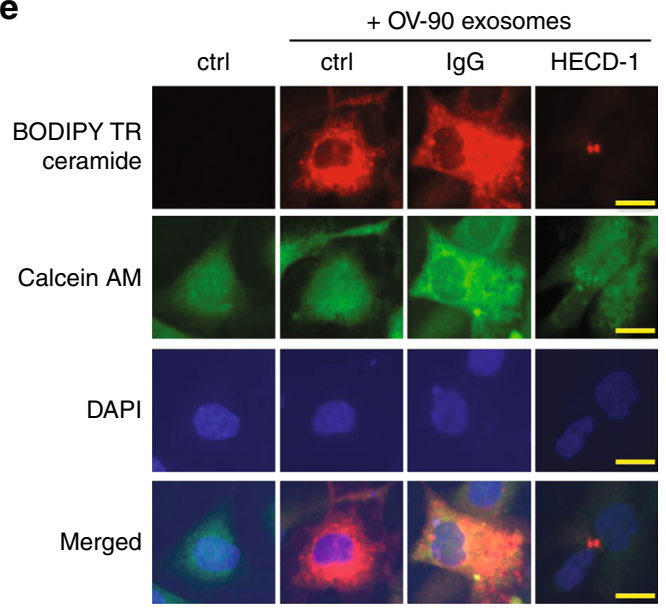

Fig. $3 \mathrm{sE}$-cad-positive exosomes promote angiogenesis in vitro and in vivo. a Cell migration assay of HUVEC treated with exosomes (25 $\left.\mu \mathrm{g} \mathrm{mL}{ }^{-1}\right)$ in the presence or absence of E-cadherin neutralizing antibodies, HECD-1 $\left(100 \mu \mathrm{g} \mathrm{mL}^{-1}\right)$. b Permeability analysis of HUVEC measured by the percentage change of FITC-dextran flux (excitation $485 \mathrm{~nm}$, emission $535 \mathrm{~nm}$ ) treated with exosomes $\left(25 \mu \mathrm{g} \mathrm{mL}{ }^{-1}\right.$ ) in the presence or absence of E-cadherin neutralizing antibodies, HECD-1 $\left(100 \mu \mathrm{g} \mathrm{mL}^{-1}\right)$. c Tube formation assay of HUVEC treated with exosomes $\left(25 \mu \mathrm{g} \mathrm{mL}^{-1}\right)$ in the presence or absence of E-cadherin neutralizing antibodies, HECD-1 (100 $\left.\mu \mathrm{g} \mathrm{mL}^{-1}\right)$. Left: Representative images of HUVEC tube formation assay. Right: Quantification of the percentage change of the number of branching points. Bar, $100 \mu \mathrm{m}$. d In vivo Matrigel plug implant model using C57/BL6 mice subcutaneously injected with Matrigel containing exosomes $\left(25 \mu \mathrm{g} \mathrm{mL}^{-1}\right)$ in the presence or absence of E-cadherin neutralizing antibodies, HECD-1 (100 $\mu \mathrm{g} \mathrm{mL} \mathrm{m}^{-1}$ ). In vivo neovascularization is measured by the Drabkin's reagent kit 7 days after injection. Upper: Representative images of excised Matrigel plug. Lower: Quantification of the percentage change of hemoglobin content. Bar, $5 \mathrm{~mm}$. e HUVECs were treated with exosomes $\left(25 \mu \mathrm{g} \mathrm{mL}{ }^{-1}\right)$ in the presence or absence of E-cadherin neutralizing antibodies, HECD-1 $\left(100 \mu \mathrm{g} \mathrm{mL}^{-1}\right)$. Red: BODIPY TR ceramide; Green: Calcein AM; Blue: DAPI. Bar, $5 \mu \mathrm{m}$. For in vivo Matrigel plug model, $n=6$ per group, and were conducted twice. For the other assays, $n=3$ per group, all experiments were repeated three times. Error bar indicates SD of the mean. ${ }^{\star} P<0.05,{ }^{\star \star} P<0.01$ versus untreated control using one-way analysis of variance followed by Tukey's least significant difference post hoc test 
The source of sE-cad in ovarian cancer is unclear: it can be generated by ectodomain shedding, and it is likely to involve more than one source. Our results suggest that sE-cad may be arising from the tumor itself. Similar observations were reported previously ${ }^{9}$. Our findings are the first to demonstrate sE-cad cleavage from exosomes, adding E-cadherin as a new member of exosomal protein and suggesting that sE-cad may play a role in the crosstalk between the tumor and the tumor microenvironment that supports disease progression. Of interest, intact other than the cleaved form of E-cadherin was found on exosomes. Indeed, intact E-cadherin may be more advantageous compared with the soluble protein as the exosomal membrane, which is rich in cholesterol and sphingomyelin, helps to maintain a stable conformation of the cell surface protein for a half-life that is

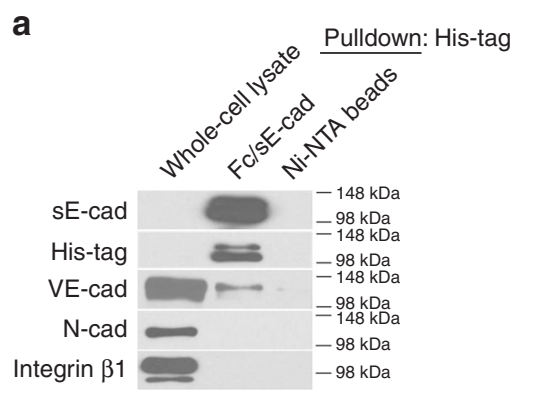

b

C
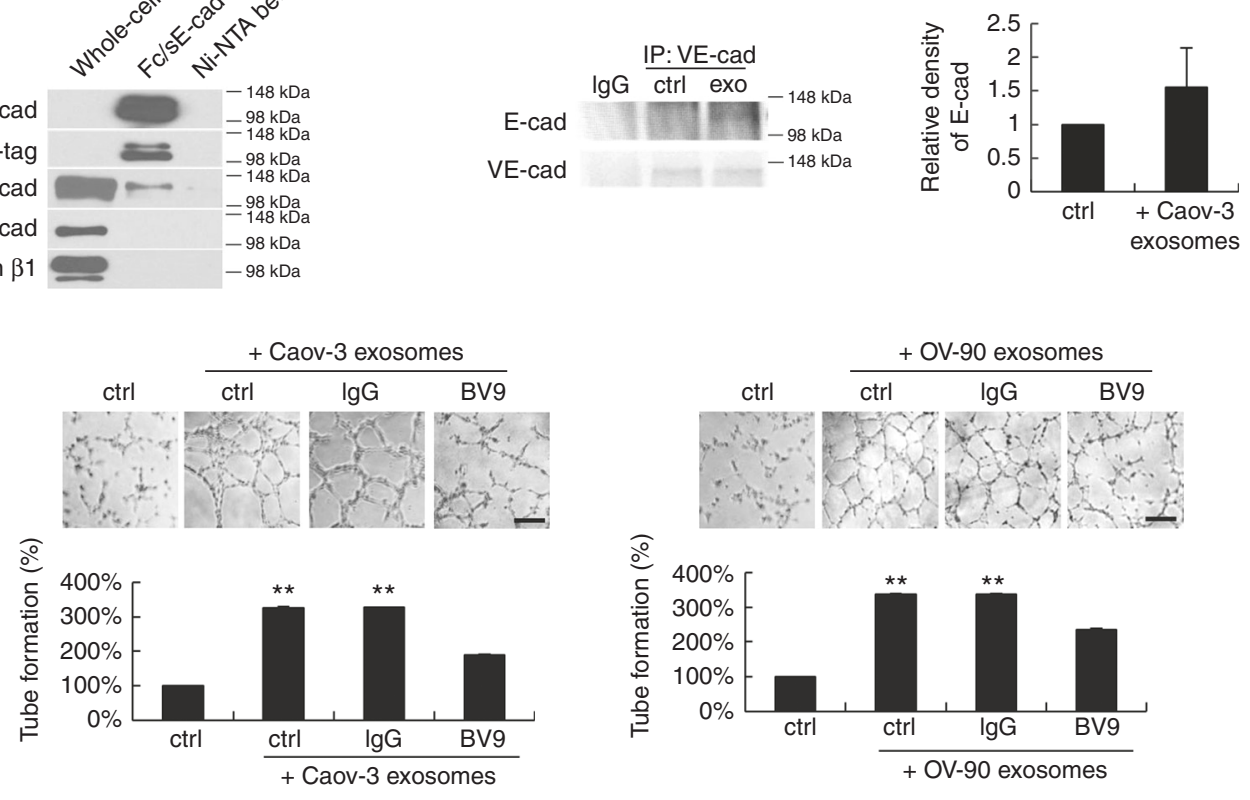

d

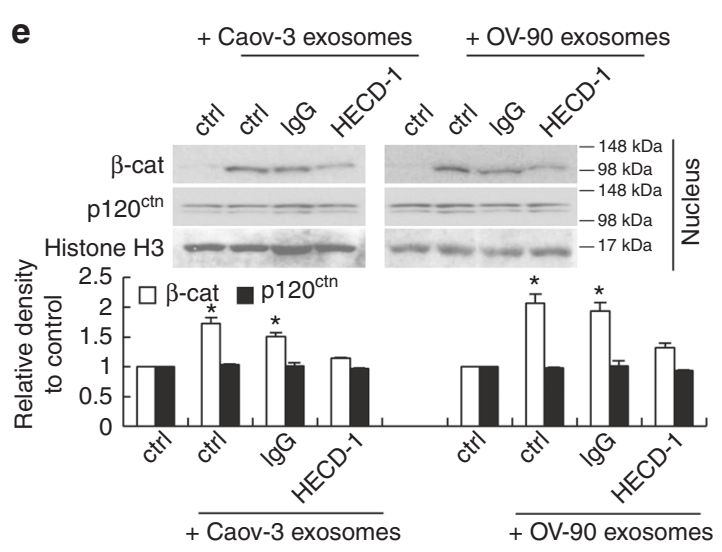

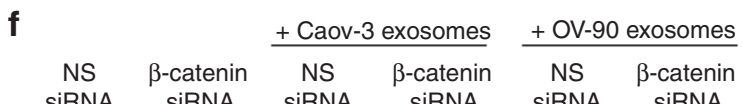

g
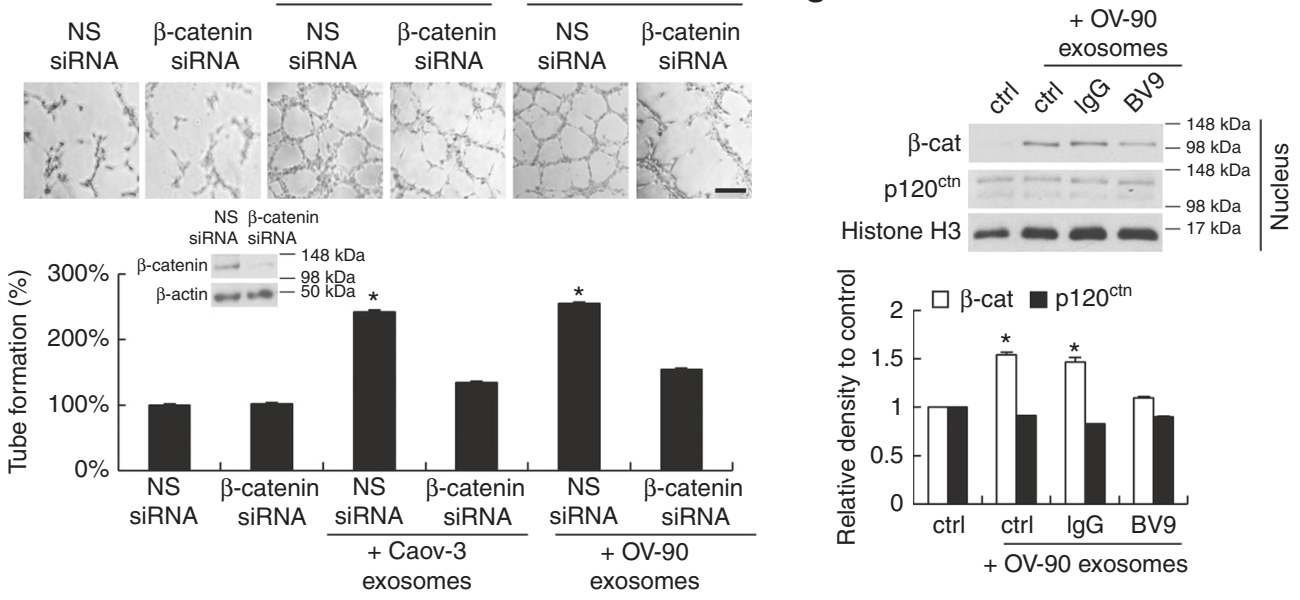
much longer (in some cases it can be as long as 90 days) compared to the soluble protein (from minutes to hours), thus enabling it to travel over long distance and displays significantly more effective cancer phenomena ${ }^{31,32}$.

$\mathrm{sE}$-cad-positive vesicles were also observed in the ascites of ovarian carcinoma patients. Several observations could be made: (a) ascites-derived exosomes had similar density features as exosomes secreted by human ovarian cancer lines; (b) sE-cadpositive exosomes in the ascitic fluid induce angiogenesis, and (c) selective targeting of sE-cad in vivo reduces ascites formation. Indeed, tumor-derived exosomes are commonly found in the ascites of ovarian cancer patients. Although mechanisms have not been fully elucidated, the cell surface molecules, EpCAM and $\mathrm{CD} 24^{33}$, have been shown to exert gelatinolytic activities that may contribute to tumor spreading. Exosomes isolated from a cisplatin-resistant human ovarian cancer cell line 2008/C13*5.25 are able to export cisplatin ${ }^{34}$, and exosomes derived from tumor cells and dendritic cells are linked to anti-tumor immune responses ${ }^{35,36}$. There are also emerging evidences suggesting that tumor cell-derived exosomes could modify and initiate a premetastatic niche $e^{37,38}$.

In search of mechanisms underlying sE-cad regulation of angiogenesis, we found that sE-cad mediates heterophilic transconnection with VE-cadherin. This provides a novel and attractive mechanism whereby endothelial cells, which lack E-cadherin, can lead to angiogenic signaling. Although previously thought to interact in an exclusively homophilic manner, there is now intriguing evidence that different cadherins can be associated in heterophilic binding ${ }^{39}$. These heterophilic interactions may serve as a new paradigm of cell-cell communication ${ }^{39}$. Heterodimer cadherin complexes have been reported to form between $\mathrm{N}$ - and $\mathrm{R}$-cadherin using $\mathrm{L}$ cell transfections ${ }^{40}, \mathrm{~N}$ - and E-cadherin in endoderm-derived cells ${ }^{41}$, and L1- and E-cadherin in intestinal epithelium ${ }^{42}$. Our finding is the first to report heterodimeric E-/ VE-cadherin complex and also define a novel heterotypic connection between tumor cell and its microenvironment. Our data (Supplementary Fig. 10) and previous studies showed that $\beta$ catenin is present in exosomes ${ }^{43}$. Thus, although we cannot exclude the possibility that $\beta$-catenin originates from the exosomes in mediating the angiogenic response, our findings delineate a novel mechanism that links $\beta$-catenin and NFkB. Whereas crossregulation between $\beta$-catenin and NFKB has been generally reported ${ }^{23-25}$, we found that they were two parallel pathways. Sequential activation of $\beta$-catenin and NFKB has not been reported, but a possible interplay of $\beta$-catenin and other signaling pathway during embryonic development is recently suggested $^{44}$. The sequential activation is known to allow continuous input from the signaling pathway for sustaining transcriptional and subsequent cellular response. The combined operation of different mechanisms suggests that the interaction of sE-cad/VE-cadherin is a crucial event and emphasizes the important role of sE-cad/VE-cadherin that has in contributing to the angiogenic process.

VEGF has been considered as one of the most promising therapeutic anti-angiogenic targets ${ }^{45-50}$. Bevacizumab, a recombinant humanized monoclonal antibody that targets VEGF, has been used in the clinic and shown significant progression-free survival benefit as a single agent or in combination with front-line chemotherapy carboplatin and paclitaxel in advanced or recurrent ovarian cancer patients ${ }^{51,52}$. However, although bevacizumab is effective, it and other VEGF inhibitors also interfere with the normal VEGF pathway, leading to numerous serious adverse side effects ${ }^{53}$. The acquisition of resistance to therapy also necessitates new strategies targeting angiogenesis. We found that sE-cad possessed similar VEGF efficiency in angiogenesis, but in a VEGF-independent manner. VEGF-independent angiogenesis pathways were reported in ovarian and other cancers ${ }^{44,55}$, suggesting the existence of alternative pro-angiogenic factors and signaling molecules. Importantly, targeting sE-cad might have an advantage over VEGF, as it is less sensitive to some resistance mechanisms developed by cancer cells ${ }^{56}$. Moreover, targeting sEcad could provide the best approach not only on primary tumor angiogenesis, but also at disrupting the vascular barrier that contributes to ascites formation, the most distressing complication of ovarian cancer $^{57}$. The specific expression in tumor tissues may be hypothesized to limit adverse off-target toxicities. Recent evidence suggests that mAb DECMA-1, which preferentially reacts with the shed ectodomain sE-cad fragment as compared to intact E-cadherin on normal cells, can be exploited for tumortargeted therap $y^{58}$. However, sE-cad has only been characterized in anti-cancer cell proliferation effects. Here, we showed for the first time that sE-cad may be involved in other aspects of tumor progression, such as angiogenesis. These results reveal a new direction for understanding the oncogenic roles of sE-cad in tumor progression. High serum sE-cad level has also been detected in patients with breast, gastric, and colon cancer, and its expression positively correlates with the aggressive behavior ${ }^{59}$. We found that sE-cad containing exosomes were present and able to induce angiogenesis in MCF-7 breast and HCT116 colon cancer cells (Supplementary Fig. 11). Moreover, sE-cad-positive exosomes can also be found in the ascitic fluids of patients with colon, breast, liver, endometrial, and stomach cancer (Supplementary Table 2), suggesting that the angiogenic function of sEcad that we propose may have broader implications for other tumor types as well.

\footnotetext{
Fig. 4 Heterodimerization of sE-cad with VE-cadherin and the activation of VE-cadherin/ $\beta$-catenin signaling cascade. a His-tag pull down assay using sE$\mathrm{cad} / \mathrm{Fc}\left(10 \mu \mathrm{g} \mathrm{mL} \mathrm{m}^{-1}\right)$ on Ni-NTA beads and HUVEC lysate $(1 \mathrm{mg})$. b Immunoprecipitation assay using anti-VE-cadherin in HUVEC lysates (1 mg) untreated or treated with exosomes $\left(25 \mu \mathrm{g} \mathrm{mL}^{-1}\right)$. Left: Representative western blot images. Right: Densitometry of E-cadherin. c Tube formation assay of HUVEC treated with exosomes $\left(25 \mu \mathrm{g} \mathrm{m}^{-1}\right)$ in the presence or absence of $\mathrm{VE}$-cadherin neutralizing antibodies, BV9 $\left(10 \mu \mathrm{g} \mathrm{mL}{ }^{-1}\right)$. Upper: Representative images of HUVEC tube formation assay. Lower: Quantification of the percentage change of the number of branching points. Bar, $100 \mu \mathrm{m}$. d Subcellular fractionation of HUVEC treated with exosomes $\left(25 \mu \mathrm{g} \mathrm{mL} \mathrm{m}^{-1}\right)$ for 0,30 , or $60 \mathrm{~min}$. Upper: Representative images of nuclear fractions. Lower: Densitometry of $\beta$-catenin ( $\beta$-cat) and p120 catenin (p120ctn). e Subcellular fractionation of HUVEC treated with exosomes $\left(25 \mu \mathrm{g} \mathrm{mL}{ }^{-1}\right)$ in the presence of E-cadherin neutralizing antibodies, HECD-1 (100 $\left.\mu \mathrm{g} \mathrm{mL}^{-1}\right)$ for $60 \mathrm{~min}$. Upper: Representative images of nuclear fractions. Lower: Densitometry of $\beta$-catenin ( $\beta$-cat) and p120 catenin ( $120^{\mathrm{ctn}}$ ). f Tube formation assay of HUVEC transiently transfected with non-specific (NS) siRNA or $\beta$-catenin siRNA (20 nM) for $24 \mathrm{~h}$, treated with exosomes $\left(25 \mu \mathrm{g} \mathrm{mL}{ }^{-1}\right)$. Upper: Representative images of HUVEC tube formation assay. Lower: Quantification of the percentage change of the number of branching points. Representative western blot images of $\beta$-catenin knockdown in HUVEC were also included. Bar, $100 \mu \mathrm{m}$. $\mathbf{g}$ Subcellular fractionation of HUVEC treated with exosomes $(25 \mu \mathrm{g} \mathrm{mL}-1)$ in the presence of VE-cadherin neutralizing antibodies, BV9 $\left(10 \mu \mathrm{g} \mathrm{mL}{ }^{-1}\right)$ for $60 \mathrm{~min}$. Left: Representative images of nuclear fractions. Right: Densitometry of $\beta$-catenin ( $\beta$-cat) and $p 120$ catenin ( $120^{\mathrm{ctn}}$ ). $n=3$ per group, all experiments were repeated three times. Error bar indicates SD of the mean. ${ }^{\star} P<0.05$ versus untreated control using one-way analysis of variance followed by Tukey's least significant difference post hoc test
} 


\section{Methods}

Cell culture and patient samples. The OVCAR-3, Caov-3, and OV-90 human ovarian cancer cell lines, the MCF-7 human breast cancer cell line, and the HCT116 prostate cancer cell line were obtained from ATCC and maintained in standard conditions as described ${ }^{60}$. The MCAS and OVK18 (gifts from Dr. L. Cheung, School of Biomedical Sciences, University of Hong Kong) were maintained in MEM containing 20\% FBS and RPMI containing 5\% FBS, respectively. TOV21G and TOV112D (gifts from Dr. D. Chan, Department of Obstetrics and Gynecology, University of Hong Kong) were maintained in M199:MCDB105 containing 15\% FBS. HUVECs and HMVECs were purchased from Clonetics (San Diego, CA) and cultured in F12K supplemented with FBS (10\%), endothelial cells growth supplement $\left(20 \mu \mathrm{g} \mathrm{mL}^{-1}\right)$, heparin $\left(90 \mathrm{U} \mathrm{mL}^{-1}\right)$, penicillin $\left(100 \mathrm{U} \mathrm{mL}^{-1}\right)$, and streptomycin $\left(100 \mu \mathrm{g} \mathrm{mL}^{-1}\right)$. The third to eighth passages of HUVECs or HMVECs were used in these studies to ensure genetic stability of the culture. Al cells were kept in a humidified incubator with $5 \% \mathrm{CO}_{2}$ at $37^{\circ} \mathrm{C}$. Primary tumor ascitic samples were obtained from 35 ovarian cancer patients and 15 patients with various other cancer types. Peritoneal fluids were obtained from six patients operated from conditions other than cancer. Normal human OSE were derived from surface scrapings of normal ovaries from women with nonmalignant gynecological diseases and FTE were obtained from patients undergoing bilateral salpingo-oophorectomies. OSE and FTE were cultured in M199:

MCDB105 supplemented with 15\% FBS. The use of these specimens was approved

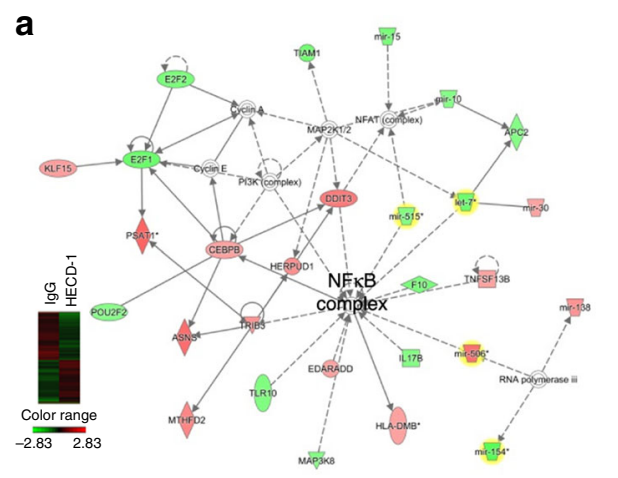

C

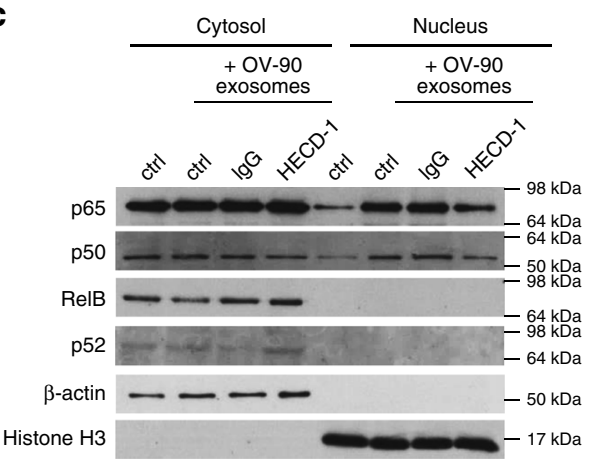

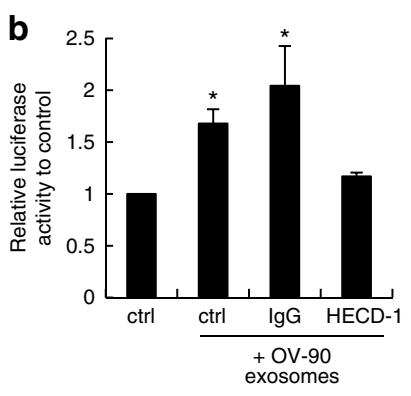

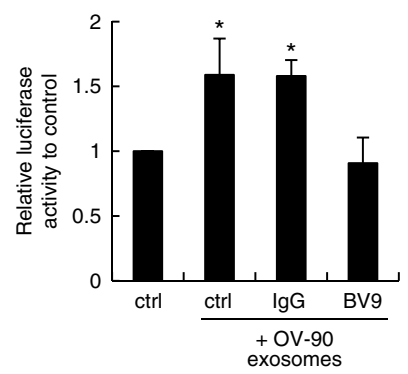

d
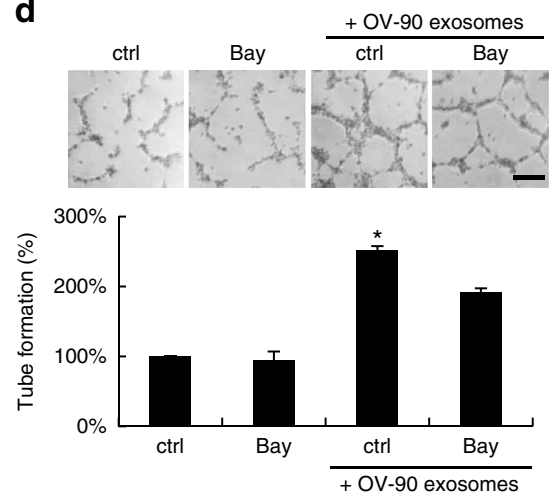

$\mathbf{e}$
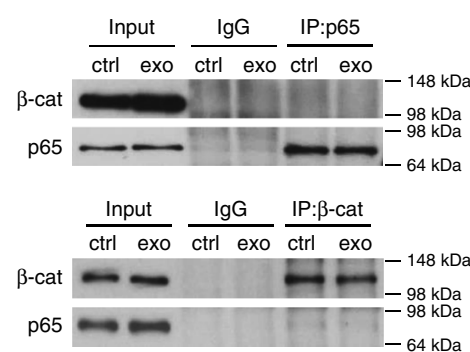

g
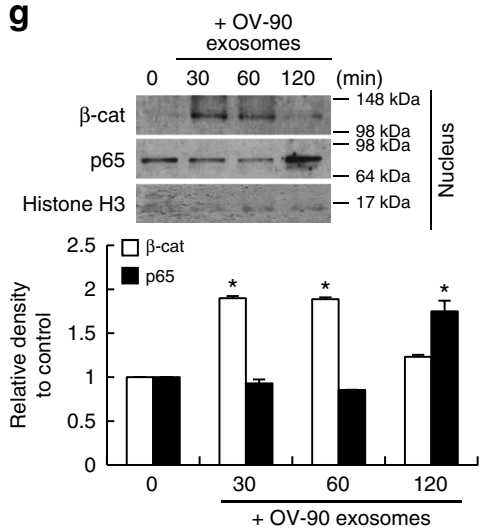
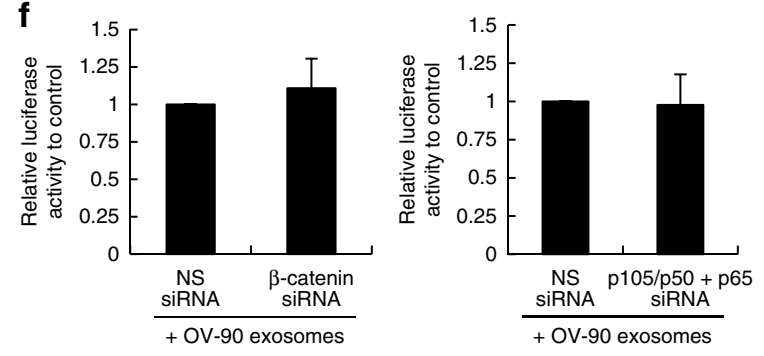

h
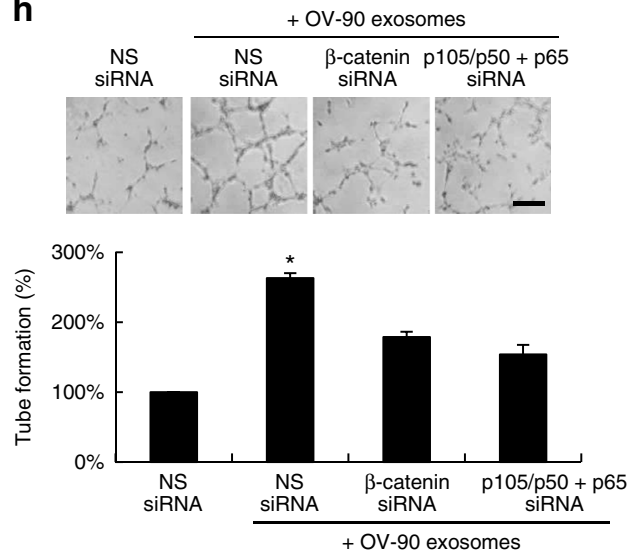
by the Institutional Ethical Review Board for Research on the use of human subjects at the University of Hong Kong.

Conditioned medium and immunodepletion. Cells were starved in serum-free medium for $24 \mathrm{~h}$ after washing with PBS twice. Conditioned medium were collected, centrifuged at $1000 \mathrm{~g}$ for $5 \mathrm{~min}$ and filtered through a $0.2 \mu \mathrm{m}$ filter. Immunodepletion of sE-cad was performed by incubating the conditioned medium with $100 \mu \mathrm{g} \mathrm{mL} \mathrm{m}^{-1} \mathrm{HECD}-1$ antibodies (Invitrogen, Carlsbad, CA) overnight at $4{ }^{\circ} \mathrm{C}$ with shaking. The antibodies-protein complex was removed by incubating with protein A/G PLUS agarose beads (Santa Cruz Biotechnology, Santa Cruz, CA).

Cytotoxicity assay. Cell survival was determined using the MTT assay according to the manufacturer's instructions (Sigma) and as previously described ${ }^{61}$. Briefly, cells were incubated with MTT solution for $4 \mathrm{~h}$ at $37^{\circ} \mathrm{C}$, medium was then aspirated and dissolved in $250 \mu \mathrm{L}$ DMSO. The colorimetric analysis was measured at $570 \mathrm{~nm}$ with a microplate reader (Bio-Rad).

Migration assay. HUVECs or HMVECs $\left(3 \times 10^{4}\right)$ were seeded onto $8 \mu \mathrm{m}$ pore Transwell inserts (Millipore) in a 24-well plate. Cell migration was examined $24 \mathrm{~h}$ after conditioned media or exosomes and/or HECD-1 treatment and stained with $0.5 \%$ crystal violet.

In vitro permeability assay. HUVECs or HMVECs $\left(3 \times 10^{4}\right)$ were seeded onto Transwells $(0.4 \mu \mathrm{m}$ pore; Millipore). After the endothelial monolayer formed, conditioned media or exosomes and/or HECD-1 was treated. FITC-dextran $(1 \mu \mathrm{g}$ $\mu \mathrm{L}^{-1}$; Sigma, St. Louis, $\mathrm{MO}$ ) was added to the upper chamber and the presence of FITC-dextran in the lower chamber after $20 \mathrm{~min}$ was determined by fluorescent measurement (Victor X3-PerkinElmer)

Tube formation assay. The tube formation assay was performed as described previously ${ }^{62}$. HUVECs or HMVECs $\left(1 \times 10^{5}\right)$ were seeded in a growth factorreduced Matrigel-coated 24-well plate. Cells were untreated or treated with conditioned media or exosomes in the absence or presence of E-cadherin (HECD-1, Invitrogen, Carlasbad, CA), VE-cadherin (clone BV9, Abcam, Cambridge, MA) or VEGF-neutralizing antibodies (clone 26503, R\&D System, Minneapolis, MN) or NFkB inhibitor (Bay11-7082, Sigma, St. Louis, MO) for $6 \mathrm{~h}$ at $37^{\circ} \mathrm{C}$. Images were captured under phase contrast microscopy $(\times 10)$ using a CCD camera. Twelve microscopic fields were randomly selected for each well, and the number of branch points of the tubes per field was counted.

In vivo Matrigel plug assay. Growth factor-reduced Matrigel (BD Biosciences) mixed with Fc/sE-cad or sE-cad-positive exosomes in the absence or presence of HECD-1 blocking antibodies were injected subcutaneously into 6-week-old C57/ BL6 mice. On day 7, the mice were sacrificed and Matrigels were excised to assess the angiogenic response. The formation of neovessels was indirectly determined by measuring the hemoglobin content using the Drabkin's reagent kit (Sigma, St. Louis, MO) according to the manufacturer's protocol. Six mice were used as a group and the experiment was done in duplicate.

Subcellular fractionation. Subcellular fractionation was performed as described previously ${ }^{63}$. Briefly, cells were lysed in lysis buffer containing $10 \mathrm{mM}$ HEPES, 10 $\mathrm{mM} \mathrm{NaCl}, 1 \mathrm{mM} \mathrm{KH} \mathrm{PO}_{4}, 5 \mathrm{mM} \mathrm{NaHCO} 3,5 \mathrm{mM}$ EDTA, $1 \mathrm{mM} \mathrm{CaCl}_{2}, 0.5 \mathrm{mM}$ $\mathrm{MgCl}_{2}$, and supplemented with protease inhibitors as described previously. After incubating on ice for $5 \mathrm{~min}$, cells were homogenized and tonicity was restored by adding $2.5 \mathrm{M}$ sucrose. Homogenate was centrifuged at $6300 \mathrm{~g}$ for $5 \mathrm{~min}$ at $4{ }^{\circ} \mathrm{C}$. The resulting pellet was resuspended in TSE buffer containing $10 \mathrm{mM}$ Tris, $300 \mathrm{mM}$ sucrose, $1 \mathrm{mM}$ EDTA, 0.1\% IGEPAL-CA 630 (v/v), pH 7.5, and further lysed with a homogenizer. This homogenate was subjected to centrifugation at $4000 \mathrm{~g}$ for $5 \mathrm{~min}$ the resulting pellet was repeatedly washed in TSE buffer. The final pellet, which is the nucleus, was resuspended in TSE buffer.

Sucrose density gradient fractionation. Cells were homogenized in buffer containing $10 \mathrm{mM}$ Tris-HCl, $1 \mathrm{mM}$ EDTA, pH 7.4. Homogenate or pelleted vesicles were loaded onto a step gradient comprising layers of $2,1.3,1.16,0.8,0.5$, and 0.25 $\mathrm{M}$ sucrose as described by Gutwein et al. ${ }^{64}$. The gradients were centrifuged at $100,000 \mathrm{~g}$ for $2.5 \mathrm{~h}$. Twelve fractions were collected from the top of the gradient. Samples were recovered by chloroform/methanol precipitation $(1: 4, \mathrm{v} / \mathrm{v})$ and analyzed by SDS PAGE and western blotting as described below. The amount of exosomal protein was quantified by DC Protein Assay (Bio-Rad). The particle size and concentration were estimated with Nanoparticle Tracking Analysis (ZetaView)

Exosomes isolation and characterization. Exosomes were isolated as described previously ${ }^{65}$. Briefly, conditioned media or patients' ascitic or peritoneal fluids were subjected to differential centrifugation $(300 \mathrm{~g}$ for $10 \mathrm{~min}, 2000 \mathrm{~g}$ for $10 \mathrm{~min}$, and $10,000 \mathrm{~g}$ for $30 \mathrm{~min})$. The resulting supernatant was filtered through a $0.2 \mu \mathrm{m}$ filter. Filtered supernatant was ultracentrifuged at $100,000 \mathrm{~g}$ for $70 \mathrm{~min}$. Pellet was washed with PBS and further centrifuged at $100,000 \mathrm{~g}$ for $70 \mathrm{~min}$. The resulting pellet consisting of exosomes was resuspended in PBS and stored at $-80^{\circ} \mathrm{C}$ for further analysis. For patient-derived exosomes, sE-cad level "high $(+++)$, medium $(++)$ low (+), and none (-)" refer to $>20 \mu \mathrm{g} \mathrm{m}^{-1}, 10-20 \mu \mathrm{g} \mathrm{mL}^{-1},<10 \mu \mathrm{g} \mathrm{mL}^{-1}$, and $0 \mu \mathrm{g} \mathrm{mL}^{-1}$, respectively.

Electron microscopy. Electron microscope examination of exosomes was carried out by depositing fixed exosomes onto a Formvar-carbon-coated electron microscope grid for $20 \mathrm{~min}$. For immunoelectron labeling, the grid was incubated with HECD-1 or CD63 antibodies, followed by mouse secondary antibodies conjugated with $10 \mathrm{~nm}$ gold particles. The grid was washed in PBS and fixed in $2 \%$ glutaraldehyde (Electron Microscopy Sciences) for $5 \mathrm{~min}$. The grid was then washed with water and contrasted in uranyl oxalate (Electron Microscopy Sciences) for $5 \mathrm{~min}$. Samples were then embedded in uranyl acetate and methyl cellulose for $10 \mathrm{~min}$ on ice. Air-dried grids were visualized using a Philips CM 100.

Exosomes internalization. Exosomes were labeled with $10 \mu \mathrm{M}$ BODIPY TR ceramide (Invitrogen) according to the manufacturer's instruction. Excess dyes were removed with an exosomes spin column (MW 3000) (Invitrogen). In addition, to control for incomplete dye removal, we columned purified dye in the absence of sE-cad-positive exosomes. HUVECs $\left(5 \times 10^{4}\right)$ were labeled with $2.5 \mu \mathrm{g} \mathrm{mL}{ }^{-1} \mathrm{Cal}-$ cein AM (Invitrogen). Labeled exosomes $\left(25 \mu \mathrm{g} \mathrm{mL}^{-1}\right)$ were then added to HUVECs for $24 \mathrm{~h}$. Cells were washed with PBS twice and fixed with $4 \%$ paraformaldehyde, the nuclei were counter-stained with 4',6-diamidino-2-phenylindole and mounted with Vectashield (Vector Laboratories, Burlingame, CA). The localization of fluorescent signals was determined by fluorescent microscopy (Nikon, Tokyo, Japan).

Western blot analysis. Cells were lysed with buffer containing $260 \mathrm{mM}$ Tris- $\mathrm{HCl}$, $\mathrm{pH} 6.8,0.8 \% \mathrm{SDS}(\mathrm{w} / \mathrm{v})$, and $40 \%$ glycerol, supplemented with protease inhibitors: $1 \mu \mathrm{g} \mathrm{mL}^{-1}$ aprotinin, $1 \mu \mathrm{g} \mathrm{mL}^{-1}$ leupeptin, $1 \mu \mathrm{gL}^{-1}$ pepstatin, and $1 \mathrm{mM}$ phenylmethyl sulfonyl. Equal amounts of protein $(20 \mu \mathrm{g})$ were resolved by SDS-PAGE for blotting using anti-HECD-1 (13-1700, 1:1000) (Invitrogen); anti-E-cadherin (610182, 1:1000), anti-Hsp70 (610607, 1:1000), anti-Tsg101 (612696, 1:1000), antinucleoporin p62 (610497, 1:1000), anti-cytochrome c (556433, 1:1000), antiGRP78 (610978, 1:1000), anti-p120 catenin (610134, 1:1000), and anti- $\beta$-catenin

Fig. $5 \mathrm{sE}$-cad-positive exosomes activate the NFKB pathway. a Top network identified by IPA. Gene signatures of HUVEC incubated with IgG pre-treated exosomes and HECD-1 pre-treated exosomes. $\mathbf{b}$ NFKB reporter assay of HUVEC treated with exosomes $\left(25 \mu \mathrm{g} \mathrm{mL} \mathrm{m}^{-1}\right)$ in the presence or absence of Ecadherin neutralizing antibodies, HECD-1 $\left(100 \mu \mathrm{gL}^{-1}\right)$ or VE-cadherin neutralizing antibodies, BV9 $\left(10 \mu \mathrm{gL} \mathrm{m}^{-1}\right)$. c Western blot analysis of the subcellular localization of NFKB subunits in HUVEC treated with exosomes $\left(25 \mu \mathrm{gL}^{-1}\right)$ in the presence or absence of E-cadherin neutralizing antibodies, HECD-1 $\left(100 \mu \mathrm{g} \mathrm{mL}^{-1}\right)$. d Tube formation assay of HUVEC treated with exosomes $\left(25 \mu \mathrm{g} \mathrm{mL}{ }^{-1}\right.$ ) in the presence or absence of Bay11-7082 (Bay) (10 $\left.\mu \mathrm{M}\right)$. Upper: Representative images of HUVEC tube formation assay. Lower: Quantification of the percentage change of the number of branching points. Bar, $100 \mu \mathrm{m}$. e Coimmunoprecipitation of NFKB subunits and $\beta$-catenin ( $\beta$-cat) in HUVEC with or without exosomes treatment $\left(25 \mu \mathrm{g} \mathrm{mL}{ }^{-1}\right)$. $\mathbf{f} \mathrm{NFKB}$ reporter assay of HUVEC transiently transfected with non-specific (NS) siRNA or $\beta$-catenin ( $\beta$-cat) siRNA ( $20 \mathrm{nM})$ for $24 \mathrm{~h}$, treated with exosomes $\left(25 \mu \mathrm{g} \mathrm{mL}^{-1}\right.$ ). TCF reporter assay of HUVEC transiently transfected with NS siRNA or p105/p50 and p65 siRNA ( $20 \mathrm{nM})$ for $24 \mathrm{~h}$, treated with exosomes $\left(25 \mu \mathrm{g} \mathrm{mL} \mathrm{m}^{-1}\right.$ ). $\mathbf{g}$ Western blot analysis of the $\beta$-catenin ( $\beta$-cat) and NFKB subunits in HUVEC treated with exosomes ( $25 \mu \mathrm{gL}^{-1}$ ) for the indicated period of time. Upper: Representative images of nuclear fractions. Lower: Densitometry of $\beta$-catenin ( $\beta$-cat) and $p 65$. $\mathbf{h}$ Tube formation assay of HUVEC transiently transfected with non-specific (NS) siRNA, $\beta$-catenin siRNA, or p105/p50 siRNA and p65 siRNA (20 nM) for 24 h with or without exosomes treatment $\left(25 \mu \mathrm{g} \mathrm{mL}^{-1}\right.$ ). Upper: Representative images of HUVEC tube formation assay. Lower: Quantification of the percentage change of the number of branching points. Bar, $100 \mu \mathrm{m} . n=3$ per group, all experiments were repeated three times. Error bar indicates SD of the mean. ${ }^{\star} P<0.05$ versus untreated control using one-way analysis of variance followed by Tukey's least significant difference post hoc test 
a

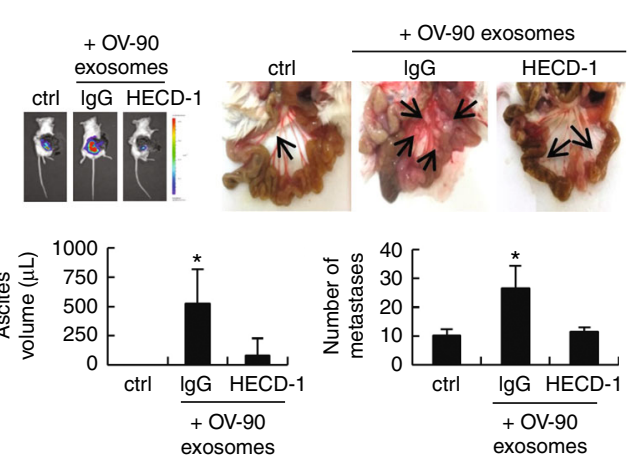

b

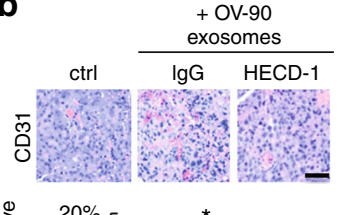

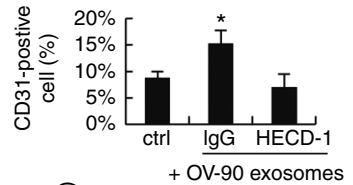
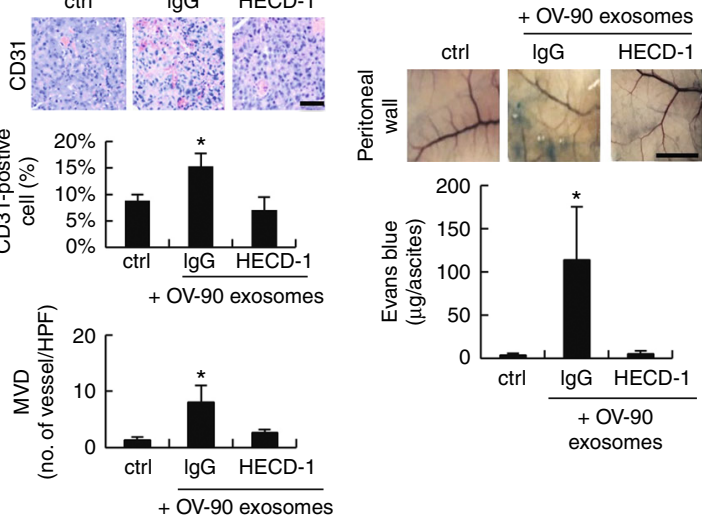

d Patient 1

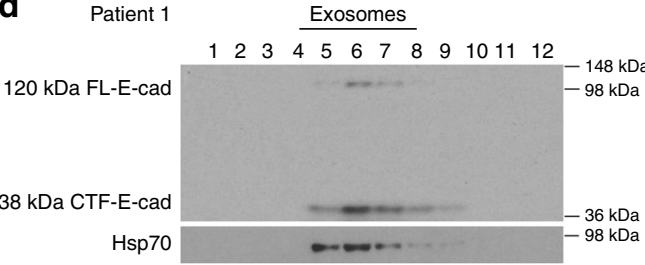

e

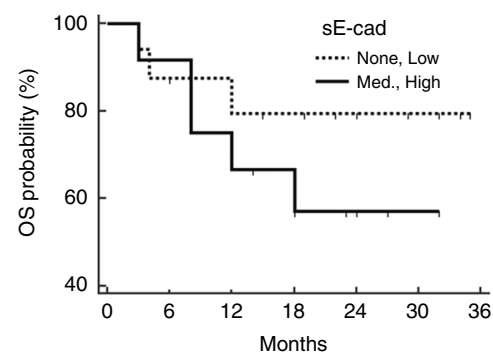

g
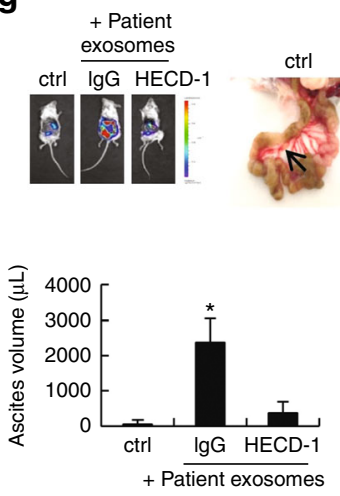

+ Patient exosomes
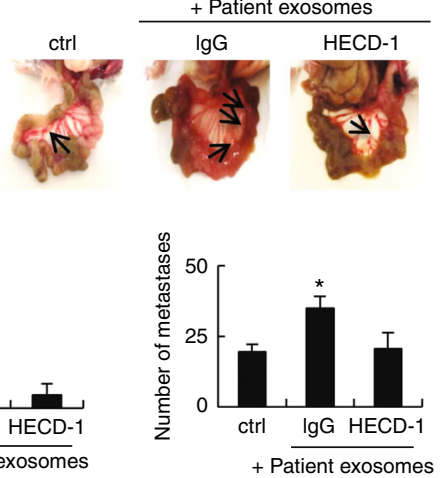

Patient 2

Exosomes

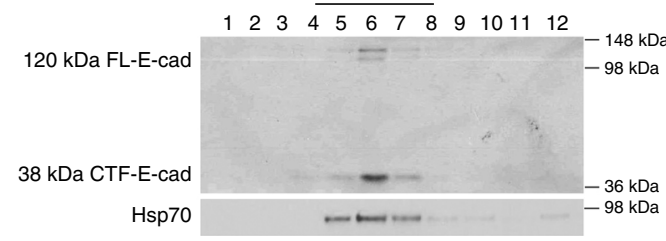

$\mathbf{f}$

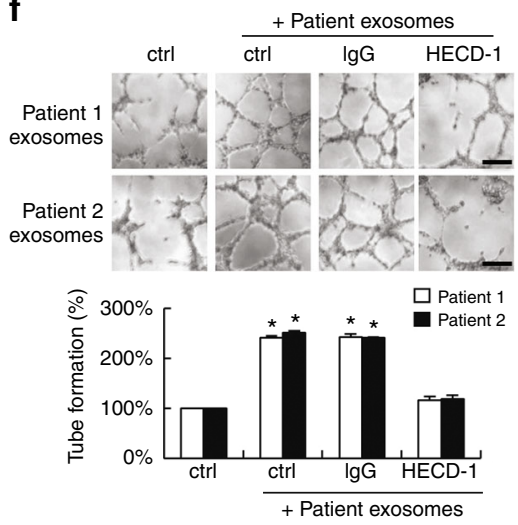

h

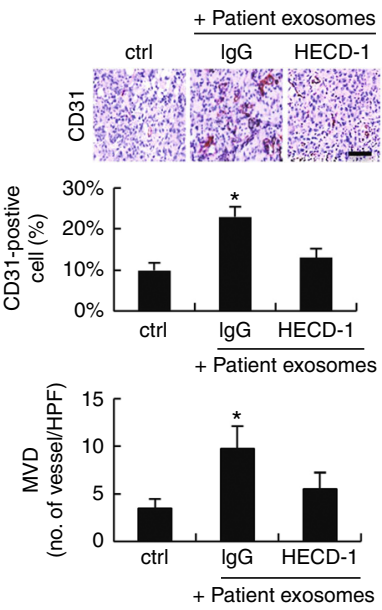

(610153, 1:1000) (BD Biosciences, San Diego, CA); and anti-CD63 (ab8219, 1:1000) (Abcam, Cambridge, MA); anti-histone H3 (9715, 1:1000), anti-VEcadherin (2500, 1:1000), anti-NFkB p65 (6956, 1:1000), anti-NFkB p105/p50 (12540, 1:1000), anti-NFkB p100/52 (4882, 1:1000), and anti-RelB (4922, 1:1000) (Cell signaling, Austin, TX); anti-integrin $\beta 1$ (MAB1965, 1:1000) (Chemicon, Billerica, MA); anti-His-tag (70796-3, 1:1000) (Novagen, Darmstadt, Germany); anti-N-cadherin (C3865, 1:1000), anti-GM130 (G7295, 1:1000), and anti- $\beta$-actin
(A5316, 1:5000) (Sigma, St. Louis, MO). The membrane was washed and incubated with appropriate secondary antibodies (1:3000) (Bio-Rad) for $1 \mathrm{~h}$. Immunoreactivities were visualized using the enhanced chemiluminescence system (Perkin Elmer, Waltham, MA). $\beta$-actin was probed to ensure equal protein loading. For analyzing conditioned medium, equal loading was determined by visualizing total protein level with Coomassie blue staining (Bio-Rad). Scans of the full blots are shown in Supplementary Figs. 12-18. 
Fig. 6 The angiogenic role of sE-cad-positive exosomes is clinically relevant. a Intraperitoneal dissemination assay using OV-90-derived exosomes. Upper left: Representative images of bioluminescence record. Upper right: metastasis in the peritoneal cavity, arrows indicate tumor. Lower: Quantification of the number of metastases and aspirated ascitic fluid. $\mathbf{b}$ Immunohistochemical staining of CD31. Upper: Representative images of immunohistochemical staining. Middle: Quantification of CD31-positive cells. Lower: Microvessel density (MVD) was expressed as number of vessels per high-power field (HPF). Bar, $50 \mu \mathrm{m}$. c NOD/SCID mice bearing HEYA8 tumors were administered with Evans blue. Upper: Representative image of Evans blue leakage in the abdominal wall. Lower: Ascites were aspirated and Evans blue content was quantified. Bar, $5 \mathrm{~mm}$. d Sucrose density gradient fractionation of exosomes isolated from ovarian cancer patients' ascites. The presence of E-cadherin is determined by western blotting. e Kaplan-Meier overall survival (OS) analysis of clinical samples of none-low or med-high sE-cad-positive exosomes expression, significance were analyzed using the log-rank test. $\mathbf{f}$ Tube formation assay of HUVEC treated with patients-derived exosomes $\left(25 \mu \mathrm{g} \mathrm{mL}^{-1}\right)$ in the presence or absence of E-cadherin neutralizing antibodies, HECD-1 $\left(100 \mu \mathrm{g} \mathrm{mL}^{-1}\right)$. Upper: Representative images of HUVEC tube formation assay. Lower: Quantification of the percentage change of the number of branching points. $\mathbf{g}$ Intraperitoneal dissemination assay using patient-derived exosomes. Upper left: Representative images of bioluminescence record. Upper right: metastasis in the peritoneal cavity, arrows indicate tumor. Lower: Quantification of the number of metastases and aspirated ascitic fluid. $\mathbf{h}$ Immunohistochemical staining of CD31. Upper: Representative images of immunohistochemical staining. Middle: Quantification of CD31-positive cells. Lower: MVD was expressed as number of vessels per HPF. Bar, $50 \mu \mathrm{m} . n=3$ per group; and the experiment was conducted at least twice. Error bar indicates SD of the mean. ${ }^{\star} P<0.05$ versus untreated control using one-way analysis of variance followed by Tukey's least significant difference post hoc test

Pull down assay. HUVEC was lysed in lysis buffer for native conditions (50 mM $\mathrm{NaH}_{2} \mathrm{PO}_{4}, 300 \mathrm{mM} \mathrm{NaCl}, 10 \mathrm{mM}$ imidazole, 1\% NP-40, pH 8.0) supplemented with protease inhibitors. Cells were homogenized and centrifuged at $14,000 \mathrm{rpm}$ for $5 \mathrm{~min}$. Supernatant $(1 \mathrm{mg})$ was then incubated with $10 \mu \mathrm{g} \mathrm{mL} \mathrm{L}^{-1} \mathrm{sE}-\mathrm{cad} / \mathrm{Fc}$ for $6 \mathrm{~h}$ on a shaker at $4{ }^{\circ} \mathrm{C}$ to facilitate protein-protein interaction. The suspension was further incubated with Ni-NTA for $1.5 \mathrm{~h}$. Protein-beads complex was collected by centrifugation at $1000 \mathrm{~g}$ for $5 \mathrm{~min}$. Beads were washed with washing buffer $(50 \mathrm{mM}$ $\mathrm{NaH}_{2} \mathrm{PO}_{4}, 300 \mathrm{mM} \mathrm{NaCl}, 20 \mathrm{mM}$ imidazole, $\mathrm{pH}$ 8.0) for three times. Protein was eluted by incubating with elution buffer $\left(50 \mathrm{mM} \mathrm{NaH}_{2} \mathrm{PO}_{4}, 300 \mathrm{mM} \mathrm{NaCl}, 500\right.$ $\mathrm{mM}$ imidazole, $\mathrm{pH} 8.0$ ) for $15 \mathrm{~min}$. The elution product was subjected to western blot analysis.

Immunoprecipitation. Nuclear extracts were lysed in immunoprecipitation buffer (2 mM EDTA, $50 \mathrm{mM}$ Tris, $\mathrm{pH} 7.5,150 \mathrm{mM} \mathrm{NaCl}$, and 1\% NP-40) supplemented with protease inhibitors as described previously ${ }^{66}$. Equal amounts of protein $(1 \mathrm{mg})$ were precleared with protein A/G PLUS agarose beads (Santa Cruz Biotechnology) for $2 \mathrm{~h}$. Supernatant was then incubated with anti-p65 antibodies or mouse IgG at $4{ }^{\circ} \mathrm{C}$ overnight. Protein-antibodies complexes were collected by incubating with protein A/G PLUS agarose beads (Santa Cruz Biotechnology) for $4 \mathrm{~h}$. Non-specific binding was removed by washing with immunoprecipitation buffer. Final products were eluted with sample buffer (125 mM Tris, pH 6.8, $20 \%$ glycerol, $1.6 \% 2$ mercaptoethanol, and $1 \%$ SDS) and subjected to western blot analysis.

siRNA transfection. $\beta$-catenin-specific siRNA (J-003482-09), p105/p50-specific siRNA (D-003520-01), and p65-specific siRNA (D-003533-03) were purchased from Dharmacon (Lafayette, CO). A non-targeting siRNA was used as control (D001210-01). Cells $\left(2.5 \times 10^{5}\right.$ per well $)$ were seeded in 6-well plates and transfected overnight with $20 \mathrm{nM}$ siRNA using siLentFect (Bio-Rad) according to the manufacturer's protocol.

Microarray analysis. Total RNA was extracted with TRIzol reagent (Invitrogen) according to the manufacturer's protocols. Briefly, the RNA was quantified with NanoDrop1000 and its quality was checked with an Agilent 2100 Bioanalyzer. 500 ng of total RNA were amplified and labeled to generate biotin-labeled cDNA using the GeneChip WT PLUS Reagent kit and then hybridized onto the GeneChip Human Gene 2.0 ST Array using the GeneChip Hybridization kit (Affymetrix), according to the manufacturer's protocols. After each array was washed and stained using the Fluidics Station 450, CEL files were generated by scanning with the GeneChip Scanner 7G with Command Console ${ }^{\circledast}$ (AGCC) software. The array quality was assessed with the Expression Console software (version 1.4). The data were normalized with the robust multiarray averaging (RMA) method using the Partek Genomics Suite 6.6. An expression ratio of 1.5 was chosen to define genes that are differentially expressed. IPA (Ingenuity Systems) was used to generate networks and assess statistically-relevant functions and pathways.

Luciferase reporter assay. Briefly, cells $\left(2.5 \times 10^{5}\right.$ per well $)$ were seeded in 6 -well plates and transfected overnight with $1 \mu \mathrm{g}$ of NFkB, TOPLASH, or FOPFLASH reporter construct (Clontech, Palo Alto, CA) and cotransfected with $0.5 \mu \mathrm{g}$ of $\beta$ galactosidase expression plasmid (pSV- $\beta$-gal; Promega, Madison, WI), using Lipofectamine 2000 (Life Technologies). $24 \mathrm{~h}$ after transfection, cells were treated with exosomes with or without HECD-1 blocking antibodies for $6 \mathrm{~h}$, and then harvested with reporter lysis buffer (Promega). Luciferase activity was assayed using the luciferase assay kit according to the manufacturer's protocol (Promega). $\beta$-galactosidase activity was used to normalize the transfection.

Orthotopic in vivo ovarian cancer model. All mouse studies were performed in accordance with protocols approved by the University of Hong Kong Animal Care and Use Committee. Female NOD/SCID mice were purchased from the Charles River Laboratories and maintained as previously described ${ }^{60}$. HEYA8 $\left(1 \times 10^{6}\right)$ were transduced with lentivirus encoding luciferase and used for intraperitoneal injection into NOD/SCID mice ( $n=3$ mice per group), and the experiment was conducted twice. OV-90 ( $25 \mu \mathrm{g}$ per dose) or ovarian cancer patient-derived exosomes $(15 \mu \mathrm{g}$ per dose) pre-treated with IgG or HECD-1 were given intraperitoneally twice a week over a period of 21 days. The mice were imaged once weekly for bioluminescence signal using the Xenogen IVIS system. At the time of sacrifice, the volume of ascites was determined with a pipette and the number of all visible $(>0.1 \mathrm{~cm})$ metastatic nodules in the peritoneal cavity was counted. Tissue specimens were fixed with formalin. The number of blood vessels was stained for CD31 (1:50 dilution, Abcam, Cambridge, MA) and counted in five random fields at 200x magnification. All staining was quantified by two investigators in a blinded fashion. To assess the extent of vascularization, counting was performed in the three highest MVD areas at high power $(200 \times)$. To measure vascular leakiness, Evans blue $(80$ $\mathrm{mg} \mathrm{kg}^{-1}$ ) (Sigma, St. Louis, MO) were injected through the tail vein and circulated for $40 \mathrm{~min}$. Evans blue dye was extracted from the ascitic fluid and its content was quantified spectrophotometrically at $595 \mathrm{~nm}$.

Statistical analysis. Data were expressed as the mean \pm SD of at least three independent experiments. Differences between control and treatment were compared for significance using the Student's test. Statistical analyses of three or more groups were compared using one-way analysis of variance (ANOVA) followed by Tukey's least significant difference post hoc test (GraphPad, San Diego, CA). $P<0.05$ was considered statistically significant. Survival estimates were computed using the Kaplan-Meier plot, and comparisons between none-low and med-high groups were analyzed using the log-rank test.

Data availability. The GEO accession number for the microarray data reported in this paper is GSE95174. All other remaining data are available within the Article and Supplementary Files, or available from the authors upon request.

\section{Received: 22 March 2017 Accepted: 18 May 2018}

Published online: 11 June 2018

\section{References}

1. Sieczkiewicz, G. J., Hussain, M. \& Kohn, E. C. Angiogenesis and metastasis. Cancer Treat. Res. 107, 353-381 (2002).

2. Schmitt, J. \& Matei, D. Targeting angiogenesis in ovarian cancer. Cancer Treat Rev. 38, 272-283 (2012).

3. Eskander, R. N. \& Tewari, K. S. Emerging treatment options for management of malignant ascites in patients with ovarian cancer. Int. J. Women's Health $\mathbf{4}$, 395-404 (2012).

4. Ribatti, D., Conconi, M. T. \& Nussdorfer, G. G. Nonclassic endogenous novel regulators of angiogenesis. Pharmacol. Rev. 59, 185-205 (2007).

5. Jeanes, A., Gottardi, C. J. \& Yap, A. S. Cadherins and cancer: how does cadherin dysfunction promote tumor progression? Oncogene 27, 6920-6929 (2008)

6. Wheelock, M. J., Buck, C. A., Bechtol, K. B. \& Damsky, C. H. Soluble 80-kd fragment of cell-CAM 120/80 disrupts cell-cell adhesion. J. Cell. Biochem. 34, 187-202 (1987)

7. De Wever, O. et al. Soluble cadherins as cancer biomarkers. Clin. Exp. Metastasis 24, 685-697 (2007). 
8. Symowicz, J. et al. Engagement of collagen-binding integrins promotes matrix metalloproteinase-9-dependent E-cadherin ectodomain shedding in ovarian carcinoma cells. Cancer Res. 67, 2030-2039 (2007).

9. Marques, F. R., Fonsechi-Carvasan, G. A., De Angelo Andrade, L. A. \& Böttcher-Luiz, F. Immunohistochemical patterns for alpha- and beta-catenin, E- and N-cadherin expression in ovarian epithelial tumors. Gynecol. Oncol. 94, 16-24 (2004).

10. Chung, A. S. \& Ferrara, N. Developmental and pathological angiogenesis. Annu. Rev. Cell Dev. Biol. 27, 563-584 (2012).

11. Mitra, A. et al. In vivo tumor growth of high-grade serous ovarian cancer cell lines. Gynecol. Oncol. 138, 372-377 (2015).

12. Kipps, E., Tan, D. S. \& Kaye, S. B. Meeting the challenge of ascites in ovarian cancer: new avenues for therapy and research. Nat. Rev. Cancer 13, 273-282 (2013).

13. Arnaoutova, I. \& Kleinman, H. K. In vitro angiogenesis: endothelial cell tube formation on gelled basement membrane extract. Nat. Protoc. 5, 628-635 (2010).

14. Bourgeois, D. L., Kabarowski, K. A., Porubsky, V. L. \& Kreeger, P. K. Highgrade serous ovarian cancer cell lines exhibit heterogeneous responses to growth factor stimulation. Cancer Cell Int. 15, 112 (2015).

15. Zupanc, H. R. H., Alexander, P. G. \& Tuan, R. S. Neurotrophic support by traumatized muscle-derived multipotent progenitor cells: role of endothelial cells and vascular endothelial growth factor-A. Stem Cell Res. Ther. 8, 226 (2017).

16. Allerstorfer, S. et al. FGF5 as an oncogenic factor in human glioblastoma multiforme: autocrine and paracrine activities. Oncogene 27, 4180-4190 (2008).

17. Belotti, D. et al. Matrix metalloproteinases (MMP9 and MMP2) induce the release of vascular endothelial growth factor (VEGF) by ovarian carcinoma cells: implications for ascites formation. Cancer Res. 63, 5224-5229 (2003).

18. Passaniti, T. \& Vitolo, M. I. In Vivo Matrigel Angiogenesis Assays. Methods in Endothelial Cell Biology. 207-222 (Springer, 2004).

19. Record, M., Subra, C., Silvente-Poirot, S. \& Poirot, M. Exosomes as intercellular signalosomes and pharmacological effectors. Biochem. Pharmacol. 81, 1171-1182 (2011).

20. Li, P., Kaslan, M., Lee, S. H., Yao, J. \& Gao, Z. Progress in exosome isolation techniques. Theranostics 7, 789-804 (2017).

21. Zebrowski, B. K. et al. Markedly elevated levels of vascular endothelial growth factor in malignant ascites. Ann. Surg. Oncol. 6, 373-378 (1999).

22. Cavallaro, U. \& Dejana, E. Adhesion molecule signalling: not always a sticky business. Nat. Rev. Mol. Cell Biol. 12, 189-197 (2011).

23. Du, Q. \& Geller, D. A. Cross-regulation between Wnt and NF-kB signaling pathways. Immunopathol. Dis. Ther. 1, 155-181 (2010).

24. Deng, J. et al. Beta-catenin interacts with and inhibits NF-kappa B in human colon and breast cancer. Cancer Cell 2, 323-334 (2002).

25. Schwitalla, S. et al. Intestinal tumorigenesis initiated by dedifferentiation and acquisition of stem-cell-like properties. Cell 152, 25-38 (2013).

26. Croager, E. Tumorigenesis: stopping the leaks. Nat. Rev. Cancer 3, 635 (2003).

27. Mason, S. D. \& Joyce, J. A. Proteolytic networks in cancer. Trends Cell Biol. 21, 228-237 (2011).

28. Gho, Y. S., Kleinman, H. K. \& Sosne, G. Angiogenic activity of human soluble intercellular adhesion molecule-1. Cancer Res. 59, 5128-5132 (1999).

29. Koch, A. E., Halloran, M. M., Haskell, C. J., Shah, M. R. \& Polverini, P. J. Angiogenesis mediated by soluble forms of E-selectin and vascular cell adhesion molecule-1. Nature 376, 517-579 (1995).

30. Banda, M. J., Knighton, D. R., Hunt, T. K. \& Werb, Z. Isolation of a nonmitogenic angiogenesis factor from wound fluid. Proc. Natl. Acad. Sci. U.S. A. 79, 7773-7777 (1982).

31. Marleau, A. M., Chen, C. S., Joyce, J. A. \& Tullis, R. H. Exosome removal as a therapeutic adjuvant in cancer. J. Transl. Med. 10, 134 (2012).

32. Kalra, H. et al. Comparative proteomics evaluation of plasma exosome isolation techniques and assessment of the stability of exosomes in normal human blood plasma. Proteomics 13, 3354-3364 (2013).

33. Runz, S. et al. Malignant ascites-derived exosomes of ovarian carcinoma patients contain CD24 and EpCAM. Gynecol. Oncol. 107, 563-571 (2007).

34. Safaei, R. et al. Abnormal lysosomal trafficking and enhanced exosomal export of cisplatin in drug-resistant human ovarian carcinoma cells. Mol. Cancer Ther. 4, 1595-1604 (2005).

35. Wolfers, J. et al. Tumor-derived exosomes are a source of shared tumor rejection antigens for CTL cross-priming. Nat. Med. 7, 297-303 (2001).

36. Zitvogel, L. et al. Eradication of established murine tumors using a novel cellfree vaccine: dendritic cell-derived exosomes. Nat. Med. 4, 594-600 (1998).

37. Peinado, $\mathrm{H}$. et al. Melanoma exosomes educate bone marrow progenitor cells toward a pro-metastatic phenotype through MET. Nat. Med. 18, 883-891 (2012).

38. Costa-Silva, B. et al. Pancreatic cancer exosomes initiate pre-metastatic niche formation in the liver. Nat. Cell Biol. 17, 816-826 (2015).
39. Prakasam, A. K., Maruthamuthu, V. \& Leckband, D. E. Similarities between heterophilic and homophilic cadherin adhesion. Proc. Natl. Acad. Sci. U.S.A. 103, 15434-15439 (2006).

40. Shan, W. S. et al. Functional cis-heterodimers of N-and R-cadherins. J. Cell Biol. 148, 579-590 (2000).

41. Straub, B. K. et al. E-N-cadherin heterodimers define novel adherens junctions connecting endoderm-derived cells. J. Cell Biol. 195, 873-887 (2011).

42. Baumgartner, W. et al. Heterotypic trans-interaction of LI- and E-cadherin and their localization in plasmalemmal microdomains. J. Mol. Biol. 378, 44-54 (2008).

43. Chairoungdua, A., Smith, D. L., Pochard, P., Hull, M. \& Caplan, M. J. Exosome release of $\beta$-catenin: a novel mechanism that antagonizes Wnt signaling. J. Cell Biol. 190, 1079-1091 (2010).

44. Watanabe, $\mathrm{H}$. et al. Sequential actions of $\beta$-catenin and Bmp pattern the oral nerve net in Nematostella vectensis. Nat. Commun. 5, 5536 (2014).

45. Olson, T., Mohanraj, D. \& Ramakrishnan, S. In vivo neutralization of vascular endothelial growth factor (VEGF) vascular permeability factor (VPF) inhibits ovarian carcinoma-associated ascites formation and tumor growth. Int. J. Oncol. 8, 505-511 (1996).

46. Mesiano, S., Ferrara, N. \& Jaffe, R. B. Role of vascular endothelial growth factor in ovarian cancer: inhibition of ascites formation by immunoneutralization. Am. J. Pathol. 153, 1249-1256 (1998).

47. Byrne, A. T. et al. Vascular endothelial growth factor-trap decreases tumor burden, inhibits ascites, and causes dramatic vascular remodeling in an ovarian cancer model. Clin. Cancer Res. 9, 5721-5728 (2003).

48. Pourgholami, M. H., Yan Cai, Z., Lu, Y., Wang, L. \& Morris, D. L. Albendazole: a potent inhibitor of vascular endothelial growth factor and malignant ascites formation in OVCAR-3 tumor-bearing nude mice. Clin Cancer Res. 12, 1928-1935 (2006).

49. Mabuchi, S. et al. Maintenance treatment with bevacizumab prolongs survival in an in vivo ovarian cancer model. Clin. Cancer Res. 14, 7781-7789 (2008).

50. Huang, J. et al. Dll4 inhibition plus aflibercept markedly reduces ovarian tumor growth. Mol. Cancer Ther. 15, 1344-1352 (2016)

51. Oliver, K. E. \& McGuire, W. P. Ovarian cancer and antiangiogenic therapy: caveat emptor. J. Clin. Oncol. 32, 3353-3356 (2014).

52. Li, X., Zhu, S., Hong, C. \& Cai, H. Angiogenesis inhibitors for patients with ovarian cancer: a meta-analysis of 12 randomized controlled trials. Curr. Med. Res. Opin. 32, 555-556 (2016).

53. Chen, H. X. \& Cleck, J. N. Adverse effects of anticancer agents that target the VEGF pathway. Nat. Rev. Clin. Oncol. 6, 465-477 (2009).

54. Raimondi, C. et al. Imatinib inhibits VEGF-independent angiogenesis by targeting neuropilin 1-dependent ABL1 activation in endothelial cells. J. Exp. Med. 211, 1167-1183 (2014).

55. Mahner, S. et al. Beyond bevacizumab: an outlook to new anti-angiogenics for the treatment of ovarian cancer. Front. Oncol. 5, 211 (2015).

56. Abdullah, S. E. \& Perez-Soler, R. Mechanisms of resistance to vascular endothelial growth factor blockade. Cancer 118, 3455-3467 (2012).

57. Tamsma, J. T., Keizer, H. J. \& Meinders, A. E. Pathogenesis of malignant ascites: Starling's law of capillary hemodynamics revisited. Ann. Oncol. 12, 1353-1357 (2001)

58. Brouxhon, S. M. et al. Monoclonal antibody against the ectodomain of Ecadherin (DECMA-1) suppresses breast carcinogenesis: involvement of the HER/PI3K/Akt/mTOR and IAP pathways. Clin. Cancer Res. 19, 3234-3246 (2013).

59. David, J. M. \& Rajasekaran, A. K. Dishonorable discharge: the oncogenic roles of cleaved E-cadherin fragments. Cancer Res. 72, 2917-2923 (2012).

60. Lam, S. S. et al. Targeting estrogen-related receptor alpha inhibits epithelialto-mesenchymal transition and stem cell properties of ovarian cancer cells. Mol. Ther. 22, 743-751 (2014).

61. Chau, W. K., Ip, C. K., Mak, A. S., Lai, H. C. \& Wong, A. S. c-Kit mediates chemoresistance and tumor-initiating capacity of ovarian cancer cells through activation of Wnt/ $\beta$-catenin-ATP-binding cassette G2 signaling. Oncogene 32, 2767-2781 (2013)

62. Leung, K. W., Pon, Y. L., Wong, R. N. \& Wong, A. S. Ginsenoside-Rg1 induces vascular endothelial growth factor expression through the glucocorticoid receptor-related phosphatidylinositol 3-kinase/Akt and beta-catenin/T-cell factor-dependent pathway in human endothelial cells. J. Biol. Chem. 281, 36280-36288 (2006)

63. Guillemin, I., Becker, M., Ociepka, K., Friauf, E. \& Nothwang, H. G. A subcellular prefractionation protocol for minute amounts of mammalian cell cultures and tissue. Proteomics 5, 35-45 (2005).

64. Gutwein, P. et al. Cleavage of L1 in exosomes and apoptotic membrane vesicles released from ovarian carcinoma cells. Clin. Cancer Res. 11, 2492-2501 (2005)

65. Théry, C., Amigorena, S., Raposo, G. \& Clayton, A. Isolation and characterization of exosomes from cell culture supernatants and biological fluids. Curr. Protoc. Cell Biol. 30, 3-22 (2006). 
66. Tang, M. K. et al. BRCA1 deficiency induces protective autophagy to mitigate stress and provides a mechanism for BRCA1 haploinsufficiency in tumorigenesis. Cancer Lett. 346, 139-147 (2014).

\section{Acknowledgments}

This work is supported by Hong Kong Research Grant Council grant (781013). A.S.T.W. is a recipient of the Croucher Senior Research Fellowship.

\section{Author contributions}

M.K.S.T. conducted the experiments, and analyzed and interpreted the results. R.L.H., H.C.L., P.P.I., A.N.Y.C., K.Y.T., and H.Y.S.N. secured surgical patient samples and conducted pathological examination of patients' samples and tests for association between sE-cad and clinicopathologic variables. P.Y.K.Y. helped in in vivo angiogenesis assays and provided technical advice. A.S.T.W. designed and supervised the project. M.K.S.T. and A.S.T.W. wrote the draft manuscript and all authors reviewed the manuscript.

\section{Additional information}

Supplementary Information accompanies this paper at https://doi.org/10.1038/s41467018-04695-7.

Competing interests: The authors declare no competing interests.
Reprints and permission information is available online at http://npg.nature.com/ reprintsandpermissions/

Publisher's note: Springer Nature remains neutral with regard to jurisdictional claims in published maps and institutional affiliations.

cc (i) Open Access This article is licensed under a Creative Commons Attribution 4.0 International License, which permits use, sharing, adaptation, distribution and reproduction in any medium or format, as long as you give appropriate credit to the original author(s) and the source, provide a link to the Creative Commons license, and indicate if changes were made. The images or other third party material in this article are included in the article's Creative Commons license, unless indicated otherwise in a credit line to the material. If material is not included in the article's Creative Commons license and your intended use is not permitted by statutory regulation or exceeds the permitted use, you will need to obtain permission directly from the copyright holder. To view a copy of this license, visit http://creativecommons.org/ licenses/by/4.0/.

(C) The Author(s) 2018 